\title{
ArcheoSciences
}

Revue d'archéométrie

40 | 2016

Varia

\section{Prospections géophysiques multi-méthodes de structures portuaires antiques à Narbonne (Aude, France)}

Multi-method geophysical surveys of ancient port structures of Narbonne (Aude, France)

Vivien Mathé, Corinne Sanchez, Guillaume Bruniaux, Adrien Camus, Julien Cavero, Camille Faïsse, Marie-Pierre Jézégou, Julie Labussière et François Lévêque

\section{OpenEdition Journals}

Édition électronique

URL : https://journals.openedition.org/archeosciences/4732

DOI : 10.4000/archeosciences.4732

ISBN : 978-2-7535-5384-2

ISSN : 2104-3728

Éditeur

Presses universitaires de Rennes

\section{Édition imprimée}

Date de publication : 30 décembre 2016

Pagination : 47-63

ISBN : 978-2-7535-5382-8

ISSN : 1960-1360

Référence électronique

Vivien Mathé, Corinne Sanchez, Guillaume Bruniaux, Adrien Camus, Julien Cavero, Camille Faïsse Marie-Pierre Jézégou, Julie Labussière et François Lévêque, «Prospections géophysiques multiméthodes de structures portuaires antiques à Narbonne (Aude, France) », ArcheoSciences [En ligne] 40 | 2016, mis en ligne le 30 décembre 2018, consulté le 23 février 2022. URL : http://

journals.openedition.org/archeosciences/4732 ; DOI : https://doi.org/10.4000/archeosciences.4732 


\title{
Prospections géophysiques multi-méthodes de structures portuaires antiques à Narbonne (Aude, France)
}

\author{
Multi-method Geophysical Surveys of Ancient Port Structures of Narbonne \\ (Aude, France)
}

\author{
Vivien Mathéa, Corinne Sanchez ${ }^{\mathrm{b}}$, Guillaume Bruniaux ${ }^{\mathrm{a}}$, Adrien Camus ${ }^{\mathrm{c}}$, \\ Julien CAvero ${ }^{\mathrm{d}}$, Camille Fä̈sse ${ }^{\mathrm{e}}$, Marie-Pierre JÉzÉGou ${ }^{\mathrm{f}}$, Julie Labussıère ${ }^{\mathrm{b}}$ \\ et François LÉvÊQUE ${ }^{a}$
}

\begin{abstract}
Résumé : Depuis 2005, une équipe interdisciplinaire de recherches tente de reconstituer la topographie du port antique de Narbonne, considéré comme un des plus grands ports de l'Empire romain. Débutées en 2007, les prospections géophysiques sont un des piliers de ce travail. Elles ont dû évoluer au fil des campagnes de mesures afin de s'adapter aux contraintes du terrain, à l'évolution des résultats et à l'émergence de nouvelles problématiques. Les structures majeures du site du Castélou / Mandirac, identifié comme étant l'embouchure aménagée d'un bras sud de l'Aude, ont été repérées puis confirmées par la fouille. Deux jetées parallèles construites en milieu humide sur une longueur de plus d'1,5 km encadrent un chenal large de $50 \mathrm{~m}$ et profond de $4 \mathrm{~m}$. D’une largeur de $15 \mathrm{~m}$ à $25 \mathrm{~m}$, elles sont aménagées en chaussée dès la fin du I ${ }^{\mathrm{er}}$ siècle par l'apport de quantités considérables de matériaux et de milliers de pieux en bois qui viennent renforcer leurs berges. Les traces d'activités portuaires y sont nombreuses. Nulle part dans le monde romain, des constructions de cette ampleur pour canaliser un fleuve n'ont été mises au jour. Elles soulignent la volonté de maîtriser un milieu fluctuant afin d'assurer une stabilité et une pérennité de ce lieu d'échanges, finalement abandonné à la fin du v viècle.
\end{abstract}

\begin{abstract}
Since 2005, an interdisciplinary team of research attempts to reconstruct the topography of the ancient port of Narbonne, considered one of the largest ports in the Roman Empire. Begun in 2007, geophysical surveys are one of the pillars of this work. They have evolved over the measurement campaigns in order to adapt to the constraints of the field, the evolution of the results and the emergence of new problems. The major structures of the site Le Castélou / Mandirac, identified as fitted mouth of a southern arm of the Aude, were located then confirmed by the excavation. Two parallel jetties constructed in wetland on a length of more than $1.5 \mathrm{~km}$ border the $50 \mathrm{~m}$-wide channel ( $4 \mathrm{~m}$-depth). With a width of $15 \mathrm{~m}$ to $25 \mathrm{~m}$, they are converted into road at the end of the first century by the contribution of considerable amounts of materials and thousands of wooden piles that reinforce their banks. Traces of port activities are numerous. Nowhere in the Roman world, works of this extent to channel a river have been uncovered. They emphasize the desire to control a fluctuating environment to ensure stability and sustainability of this place of exchange, finally abandoned in the late fifth century.
\end{abstract}

${ }^{a}$ UMR 7266 LIENSs Université de La Rochelle-CNRS, 2 rue Olympe-de-Gouges, 17000 LA ROCHELLE, France. (vivien.mathe@univ-lr.fr) (guillaume. bruniaux@univ-lr.fr)(francois.leveque@univ-lr.fr)

${ }^{b}$ UMR 5140 ASM Université Montpellier 3 - CNRS - MCC, 390 avenue de Pérols, 34970 LATTES, France. (corinne.sanchez@cnrs.fr) (julie.labussiere@gmail.com)

' ADERA, 162 Avenue Albert-Schweitzer, CS 60040, 33608 PESSAC cedex - UMR 7266 LIENSs Université de La Rochelle - CNRS, 2 rue Olympede-Gouges, 17000 LA ROCHELLE, France. (adrien.camus@univ-lr.fr)

' labex TransferS, École normale supérieure, 29 rue d'Ulm, 75230 PARIS cedex 05, France. (juliencavero@gmail.com)

'Archeodunum SAS, 8 allée Michel-de-Montaigne, 31770 COLOMIERS - UMR 5140 ASM Université Montpellier 3 - CNRS - MCC, 390 avenue de Pérols, F-34970 LATTES, France. (fcamille@live.fr)

f DRASSM, 147 plage de l'Estaque, F-13016 Marseille - UMR 5140 ASM Université Montpellier 3-CNRS - MCC, 390 avenue de Pérols, F-34 970

LATTES, France. (marie-pierre.jezegou@culture.gouv.fr) 
Mots clés : Lagune, Le Castélou / Mandirac, Port antique, Prospection électromagnétique, Prospection magnétique, Sections de résistivité électrique

Keywords: Ancient harbor, Electrical resistivity tomography, Electromagnetic survey, Lagoon, Le Castélou / Mandirac, Magnetic survey

\section{INTRODUCTION}

Le port antique de Narbonne est considéré comme l'un des plus importants du monde romain en Méditerranée occidentale. Pendant l'Antiquité, Narbonne était située au carrefour de routes majeures, notamment la Via Domitia et la voie d'Aquitaine, mais également à proximité de l'embouchure du fleuve Aude. La vallée de l'Aude offrait un axe majeur de pénétration vers l'intérieur des terres, et au-delà, vers les régions de l'Atlantique. Elle était ainsi située sur la route reliant la Méditerranée aux îles Britanniques, un axe très prisé notamment pour le commerce de l'étain.

Malgré la grandeur du site, à la fin $\mathrm{du}_{\mathrm{xx}} \mathrm{e}^{\mathrm{s}}$ siècle, la topographie, l'environnement et l'organisation des structures portuaires de Narbonne n'étaient toujours que très peu renseignées. L'importance de l'activité maritime était principalement connue grâce aux auteurs antiques tels que Strabon, Posidonius ou encore Polybe, aux sources épigraphiques (mosaïque dite " des Narbonnais » sur la Place des Corporations à Ostie) et aux épaves des navires qui transitaient à proximité de Narbonne (Solier et al., 1981).

Depuis 1950, de nombreuses études ont été menées pour mieux comprendre l'évolution de la plaine alluviale située au sud de la région de Narbonne, et pour y découvrir les infrastructures portuaires antiques que l'on suppose présentes dans cette zone. Les travaux des chercheurs comme Verdeil (1970) ou Ambert (2000) ont permis de progresser sur les problématiques environnementales liées au paysage de Narbonne, sans pour autant pouvoir décrire l'environnement autour de la lagune durant l'Antiquité et ainsi comprendre les caractéristiques du port antique. Des lacunes subsistaient, notamment par le manque de découvertes archéologiques. Les travaux des archéologues et chercheurs tels Guy (1955), Solier et coll. (1981), Falguéra et coll. (2000), ont permis d'avancer plusieurs hypothèses quant à l'organisation du port de Narbonne, sans que des vestiges liés à ces hypothèses n'aient pu faire l'objet de fouilles de grande ampleur.

Depuis 2005, une équipe de recherche interdisciplinaire coordonnée par Corinne Sanchez tente de retrouver la topographie portuaire de l'ancienne capitale de la Gaule Narbonnaise. Ce travail a consisté dans un premier temps à synthétiser la masse d'informations collectées depuis près d'un siècle (Sanchez et Jézégou, 2011) puis à mener des opérations de terrain d'envergure en une dizaine de points situés en périphérie des étangs narbonnais (figure 1). Il s'avère en effet que plus qu'un port situé en un lieu unique, il s'agirait plutôt ici d'un système portuaire composé de plusieurs entités. Il apparait en effet probable que le port fluvial urbain de Narbonne soit doté d'un système d'avant-ports (Rouzaud, 1914; 1916; Guy, 1955; Solier et al., 1981) à l'image de celui de Rome. Les seuls vestiges portuaires attestés découverts avant 2005 étaient ceux de Port-La-Nautique, au nord de l'étang de Bages-Sigean (Falguéra et al., 2000). Il s'agit d'un soubassement composé de six assises en pierre de taille enceint dans un double caisson en bois et supportant un bâtiment. Toutefois ce site n'a fonctionné que durant moins d'un siècle avant d'être abandonné autour de 70 de notre ère (Falguéra et al., 2003). D’autres sites littoraux, connus alors essentiellement par la prospection, jouaient probablement aussi un rôle important dans ce système, tel celui de Saint-Martin (Guy, 1955; Sanchez et al., 2000) ou de Tintaine (Falguéra et al., 2000) à Gruissan. Cependant, compte-tenu de leurs dimensions réduites, ces quelques sites ne pouvaient expliquer à eux seuls l'importance du rôle portuaire de la ville. Il convenait donc de rechercher un site majeur, probablement en connexion directe avec le fleuve qui reliait la cité à la mer.

L'Aude coule actuellement au nord du massif de La Clape, avant de rejoindre la mer. Ce fleuve possède une branche sud, de nos jours totalement canalisée (La Robine), qui traverse Narbonne et se jetait dans l'étang de Bages-Sigean (Ambert, 1993). Les travaux récents présentés ici ont porté sur la recherche puis l'étude d'un avant-port situé à l'embouchure de ce cours sud de l'Aude. Le choix de la zone d'étude a été basée sur des photographies aériennes montrant des traces géométriques nettes interprétées comme des constructions potentielles et des traces d'un cours d'eau (Falguéra et al., 2000). La zone est une plaine alluviale marécageuse de plusieurs kilomètres carrés à proximité d'une vaste lagune. Elle regroupe deux secteurs. Au nord, le secteur de Mandirac est composé de terres agricoles et de vignes. Au sud s'étend une vaste étendue sauvage, la réserve naturelle du Castélou. C'est ici qu'au début du $\mathrm{Xx}^{\mathrm{e}}$ siècle ont été découverts des blocs de pierre équarris d'environ un mètre de long. La 


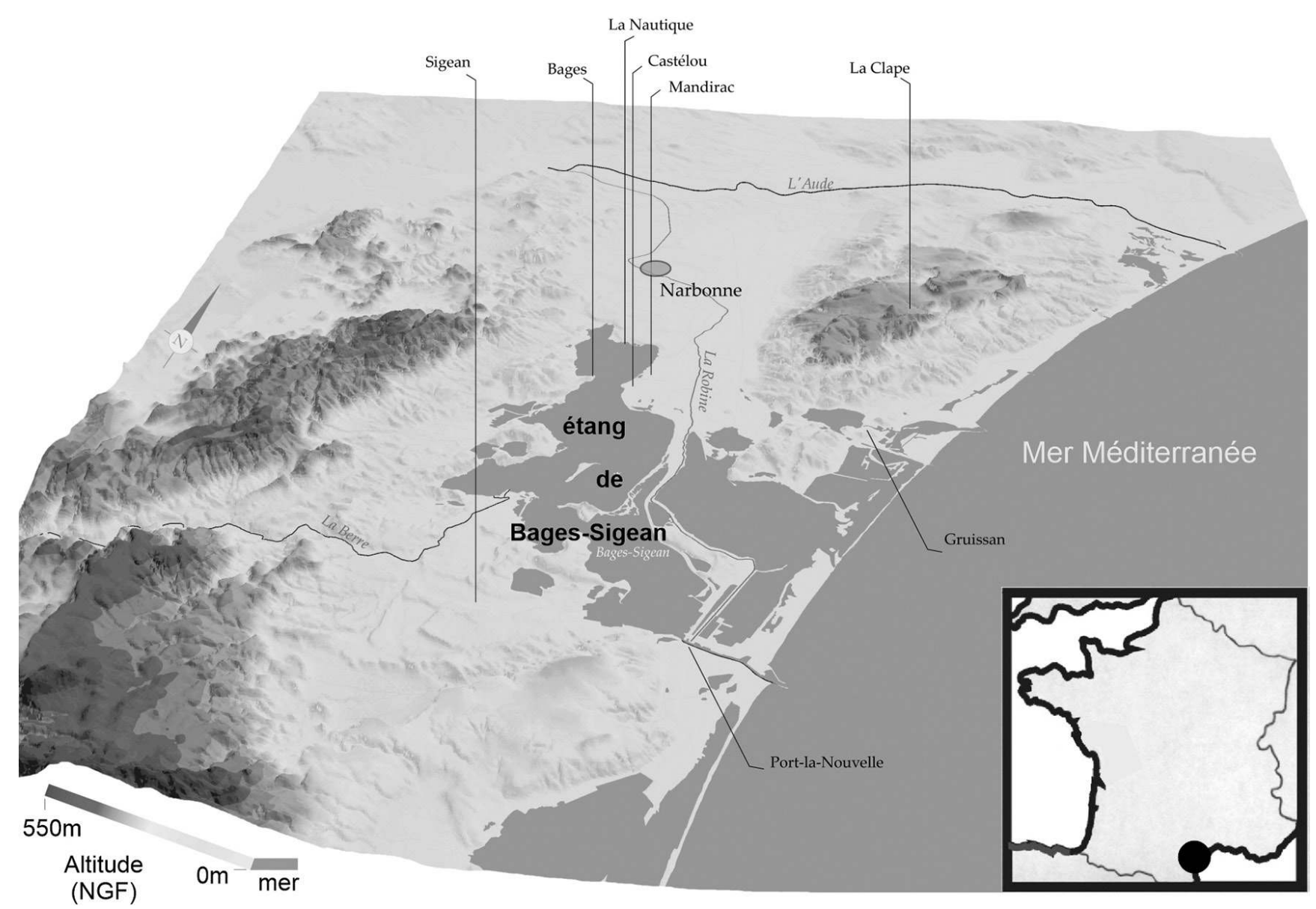

Figure 1 : Localisation du site du Castélou/Mandirac. Figure 1: Localization of the site of Castélou/Mandirac.

présence d'un quai a alors été envisagée (Rouzaud, 1914; 1916). La zone du Castélou/Mandirac est située sur la rive est de la lagune, à $6 \mathrm{~km}$ de la ville de Narbonne. Son altitude est comprise entre 0 et $2 \mathrm{~m}$ NGF. Cette zone a été largement soumise aux dynamiques fluviales et laguno-marines. L'étude des cartes anciennes a montré un changement du milieu très rapide au cours des derniers siècles (Cavero, 2010). On peut supposer qu'il en avait été de même au moins depuis l'Antiquité.

Des prospections géophysiques ont été initiées en 2007 afin de préciser les informations recueillies sur la zone du Castélou/Mandirac. L'objectif était de confirmer la présence d'un paléo-cours sud de l'Aude, de le cartographier et de rechercher des aménagements associés tels des quais, des bâtiments ou des bassins portuaires. Ces travaux se sont poursuivis les années suivantes, en étroite interaction avec les fouilles et les carottages réalisés en parallèle et dont la localisation a été le plus souvent choisie sur la base des données de prospection.
Cet article détaille les résultats des recherches géophysiques sur ce secteur confrontés aux investigations archéologiques. Il présente les divers protocoles et méthodes mis en œuvre pour s'adapter non seulement aux contraintes du terrain mais aussi aux problématiques archéologiques.

\section{Méthodes}

\section{Prospections électromagnétiques de type Slingram}

La prospection électromagnétique de type Slingram (McNeil, 1980a; Reynolds, 1997) semblait totalement adaptée pour répondre au premier objectif : rechercher et cartographier une ancienne voie d'eau au sein de la plaine alluviale. Cette méthode a en effet déjà fait ses preuves tant pour la recherche de paléochenaux (Camus et al., 2011) que pour la localisation de zones propices à l'installation de structures portuaires antiques (Mathé, 2010). 
Les prospections électromagnétiques ont été réalisées avec les conductivimètres EM31 et EM38 (Geonics Ltd). Ces deux instruments fonctionnent sur le même principe instrumental. Une bobine d'induction reliée à une source oscillante génère un champ magnétique primaire à une fréquence donnée. Lorsque le champ primaire rencontre une anomalie conductrice, un champ secondaire est créé. C'est la mesure du rapport de la composante du champ secondaire en quadrature par rapport au champ primaire qui va permettre d'identifier les hétérogénéités conductrices du sol en termes de conductivité apparente. Cette grandeur est exprimée en milliSiemens par mètre (mS/m). Jusqu’à environ $100 \mathrm{mS} / \mathrm{m}$, la composante en quadrature est directement proportionnelle au champ secondaire (McNeil, 1980b; Morris, 2009). Les valeurs faibles indiquent la présence d'accumulations de pierres, de matériaux sableux ou très secs. Les valeurs plus élevées correspondent à des milieux humides et à faible granulométrie. Sur les sols salés, de toutes les propriétés physico-chimiques qui influencent la conductivité apparente, la concentration en sels solubles est le facteur dominant (van der Lelij, 1983; Johnston et al., 1997; Mankin et Karthikeyan, 2002; Williams et al., 2006). De fortes teneurs en sels impliquent de fortes valeurs de conductivité, supérieures à $100 \mathrm{mS} / \mathrm{m}$. Dans ce cas, la composante en quadrature de phase du champ électromagnétique mesuré par le conductivimètre n'est plus proportionnelle à la conductivité du sol. La conductivité mesurée par les conductivimètres se trouve alors sous-évaluée (McNeil, 1980b) : par exemple, pour l'EM31, une mesure égale à $200 \mathrm{mS} / \mathrm{m}$ correspond à une conductivité "vraie " du sol d'environ $350 \mathrm{mS} / \mathrm{m}$. Comme l'a montré Beamish (2011), l'écart entre la mesure et la conductivité "vraie " diminue lorsque l'écartement entre la bobine émettrice et la bobine réceptrice est plus faible. C'est le cas pour l'EM38 dont l'écartement interbobines est d' $1 \mathrm{~m}$ alors qu'il est de 3,66 m pour l'EM31.

Ces deux conductivimètres se distinguent aussi par leur profondeur théorique d'investigation, comprise en mode vertical entre 3 et $6 \mathrm{~m}$ pour l'EM31, et de l'ordre de $2 \mathrm{~m}$ pour l'EM38 (McNeil, 1980a). Dans un environnement salin très conducteur, on observe une diminution de ces profondeurs d'investigation pouvant atteindre jusqu'à $50 \%$ (Callegary et al., 2007) mais en conservant un rapport signal sur bruit de la mesure acceptable (Delefortrie et al., 2014).

Chaque point de mesure est positionné par GPS avec une précision relative décimétrique et une précision absolue de l'ordre de $2 \mathrm{~m}$. L'emploi du GPS permet une grande liberté de mouvement puisqu'il évite d'implanter un carroyage. L'utilisation de cette technologie depuis le milieu des années 1990 a rendu possible la collecte rapide d'une grande quantité de données électromagnétiques (Cannon et al., 1994). L'acquisition est réalisée à raison d'une mesure toute les secondes soit une mesure tous les mètres avec une vitesse de progression de l'opérateur d'1 $\mathrm{m} / \mathrm{s}$.

Le conductivimètre EM31 est porté par l'opérateur à une hauteur comprise entre 0,8 et $1 \mathrm{~m}$ au-dessus du sol. Il permet par conséquent de progresser même dans les terrains recouverts d'une végétation haute et dense, parsemée d'arbustes, telle celle présente dans la réserve naturelle du Castélou. Par contre, son imposant volume d'investigation rend inutile une prospection à haute résolution spatiale. Les cartes ont donc été acquises par une succession de profils parallèles distants de 5 à $10 \mathrm{~m}$. À l'inverse, le conductivimètre EM38 dispose d'un volume d'investigation beaucoup plus faible donnant du sens à une prospection à plus haute résolution spatiale. Les profils ont donc été espacés d'une distance comprise entre 1 et $2 \mathrm{~m}$. Par contre, étant porté à une hauteur d'environ $0,2 \mathrm{~m}$, son usage a dû être réservé aux terrains nus ou à végétation basse. Ces instruments permettent une couverture rapide de l'espace à la résolution spatiale indiquée précédemment : 3 et 10 ha par jour et par opérateur respectivement pour l'EM38 et pour l'EM31.

\section{Sections de résistivité électrique}

Des sections de résistivité électrique ont été réalisées au sud, perpendiculairement aux prolongements probables des anomalies repérées sur les cartes de conductivité acquises plus au nord et que l'on souhaitait étudier. Elles correspondent à des pseudo-sections de la résistivité du sous-sol, comparables à des coupes. Elles permettent ainsi d'appréhender la dimension verticale de certaines structures détectées sur un plan horizontal. La résistivité, exprimée en $\mathrm{Ohm}$. mètre $(\Omega . \mathrm{m})$, est une expression de la nature et de la structure du terrain qui dépend de sa teneur en eau et en ions car c'est principalement l'eau chargée (électrolyte) contenue dans le milieu qui permet le passage du courant électrique; c'est l'inverse de la conductivité électrique. La méthode électrique est donc parfaitement adaptée à l'étude de structures bâties, résistantes, enfouies dans un milieu naturel formé de sédiments à faible granulométrie, moins résistants. Elle a été utilisée dans la zone sud du Castélou où la méthode électromagnétique s'est révélée inopérante.

Les sections ont été réalisées au moyen d'un Terrameter LS 12 voies (Abem) muni de 64 électrodes. L'acquisition repose sur l'utilisation simultanée de deux paires d'électrodes, l'une qui sert à injecter un courant électrique dans le sol en deux points de façon à créer une boucle de courant, et l'autre qui permet de mesurer la différence de potentiel entre deux autres points. Parmi les différents types de dispositifs existant, la configuration dipôle-dipôle (Reynolds, 1997) a 
été utilisée ici car elle était parfaitement adaptée à la problématique qui consiste à rechercher deux empierrements massifs bordant une bande très conductrice. Afin d'avoir une précision suffisante, dès la surface, l'écartement minimal des électrodes a été fixé à $1 \mathrm{~m}$. L'écartement maximal a été choisi de sorte à obtenir une profondeur d'investigation maximale de l'ordre de $12 \mathrm{~m}$. Les mesures ont été inversées à l'aide du logiciel Res2dinv (Geotomo Software) afin de générer, par itérations successives, un modèle représentatif des structures sédimentaires et anthropiques à l'origine des anomalies mesurées.

\section{Prospections magnétiques}

Des prospections magnétiques ont été réalisées avec pour objectif initial de cartographier d'éventuelles infrastructures portuaires là où le terrain le permettait, c'est-à-dire sur sol nu ou à végétation basse. L'instrument de mesure utilisé est un gradiomètre Ferex (Dr Foerster Institüt) muni de quatre capteurs espacés de $0,5 \mathrm{~m}$. Chaque capteur fournit directement une valeur du gradient de la composante verticale du champ magnétique exprimé en nanoTeslas par mètre $(\mathrm{nT} / \mathrm{m})$. En d'autres termes, il permet d'obtenir des cartes de répartition de la perturbation du champ magnétique terrestre générée par les matériaux présents dans le sol. Chaque capteur mesure un point tous les $0,1 \mathrm{~m}$ le long de chaque profil. Une succession d'acquisitions selon des profils parallèles permet d'obtenir 20 mesures par mètre carré, soit une densité suffisante de points pour cartographier des vestiges même de faibles dimensions situés à moins d'un mètre de la surface (Mathé et al., 2012). En effet, la profondeur d'investigation du gradiomètre dépend des dimensions des sources magnétiques et de leur aimantation par rapport à celle du milieu d'enfouissement. Cependant, par expérience, on peut admettre que la plupart des anomalies de faible intensité, telles que celles des maçonneries constituées de matériaux calcaires, proviennent d'une source située le plus souvent à quelques dizaines de centimètres sous la surface. Plusieurs caractéristiques permettent d'identifier l'origine des anomalies dipolaires : leur forme, leur intensité et le signe du pole le plus clairement exprimé. Les vestiges en pierre calcaire, peu magnétiques, sont la source d'anomalies dont le pole principal est négatif. À l'opposé, les structures fossoyées sont indiquées par des anomalies dont le pole principal est positif. Les matériaux fortement magnétiques tels que la terre cuite ou, plus encore, les morceaux de fer, sont révélés, pour leur part, par une juxtaposition d'anomalies intenses et de signe opposé.

\section{Essais au pénétromètre dynamique}

Une centaine d'essais au pénétromètre dynamique est venue compléter les prospections géophysiques. Le pénétromètre dynamique léger à énergie variable de type $\operatorname{PANDA}^{\oplus} 2$ (Sol Solution) est un outil issu de la géotechnique, souvent employé pour la reconnaissance des sols et le contrôle du compactage. Le principe consiste à enfoncer dans le sol, à coups de maillet, une tige en acier munie d'une pointe dont le diamètre est supérieur à la tige pour éviter les frottements latéraux. La tête de frappe posée sur la tige est reliée au dispositif d'acquisition de données. À chaque battage, la tête de frappe mesure la quantité d'énergie qui lui est appliquée pour enfoncer la pointe. L'enfoncement de la tige dans le sol est mesuré par l'intermédiaire d'une courroie reliant la tête au dispositif d'acquisition. Ces deux mesures entrent dans le calcul de la résistance de pointe notée Qd (s'exprimant en MégaPascal, $\mathrm{MPa}$ ) effectué via la formule mathématique dite des « Hollandais ». On obtient un profil de la résistance de pointe en fonction de la profondeur, appelé pénétrogramme. La résistance de pointe est un paramètre sensible, entre autre, à la granulométrie et à la teneur en eau du sédiment. Le pénétrogramme met donc en évidence des hétérogénéités liées à des variations des propriétés mécaniques du sol, comme sa dureté et sa compaction. Plusieurs auteurs ont ainsi détournés le pénétromètre dynamique de son usage géotechnique traditionnel pour l'appliquer à l'archéologie (Martinaud, 1999; Lenoble et Martinaud, 2003).

Deux grandeurs ont été exploitées ici. D’une part, la profondeur de refus, c'est-à-dire la profondeur à laquelle la tige s'enfonce de moins de $10^{-3} \mathrm{~m}$ suite à trois frappes de maillet. On considère alors que la progression du dispositif est stoppée par un bloc de pierre. D'autre part, la valeur de Qd, ou plus exactement sa variation, qui peut par exemple permettre de définir des entités sédimentaires qui seront mises en relation avec les résultats des sections de résistivité électrique.

\section{RÉSULTATS ET CONFRONTATION AUX VÉRIFICATIONS ARCHÉOLOGIQUES ET GÉOMORPHOLOGIQUES}

\section{Cartographie électromagnétique Slingram (2007-2010)}

Les prospections électromagnétiques de type Slingram sont les premières investigations géophysiques à avoir été mises en œuvre sur le site, essentiellement entre 2007 et 2010. Elles ont été réalisées sur une surface totale d'envi- 
ron 70 ha, centrée sur la zone ayant révélé des anomalies phytologiques visibles sur des photographies aériennes (Falguéra et al., 2000). Environ 40 ha ont été couverts par chacun des conductivimètres (EM38 et EM31) dont une dizaine d'hectares en commun sur des zones où la conductivité électrique apparente est supérieure à $200 \mathrm{mS} / \mathrm{m}$, une valeur pour laquelle la composante en quadrature de phase du champ électromagnétique mesuré par le conductivimètre n'est plus proportionnelle à la conductivité du sol. La comparaison des conductivités apparentes sur les surfaces couvertes par les deux instruments montre que les résultats issus des deux acquisitions sont étonnamment très proches. Ceci peut être attribué à plusieurs faits. D'une part, la conductivité est si élevée (plus de $100 \mathrm{mS} / \mathrm{m}$ sur les deux tiers sudouest de la surface prospectée) que les valeurs mesurées sont sous-évaluées par rapport aux conductivités " vraies ", principalement celles acquises par l'EM31 car l'écart entre la mesure et la conductivité « vraie » est plus important lorsque l'écartement entre la bobine émettrice et la bobine réceptrice est plus grand (Beamish, 2011). D'autre part, où la salinité est la plus forte, la profondeur d'investigation des conductivimètres est largement réduite. Cet effet est là aussi plus fort sur l'EM31 qui possède la profondeur théorique d'investigation la plus élevée. Et enfin, la variation de conductivité avec la profondeur est assez faible sur les zones salées, donc la conductivité apparente mesurée depuis la surface est quasiidentique malgré les profondeurs d'investigation différentes des deux instruments. Pour ces différentes raisons s'appuyant sur des travaux cités précédemment, les différences de conductivité apparente mesurées par les deux conductivimètres en un même point et sur des zones salées sont faibles. Du fait que le volume d'intégration de l'EM31 est largement supérieur à celui de l'EM38, les valeurs mesurées par l'EM31 apparaissent lissées par rapport à celles issues de l'EM38. Afin de conserver la meilleure résolution spatiale et rendre compte au mieux des hétérogénéités latérales, nous avons retenu en priorité les données mesurées à l'EM38 sur les zones ayant été prospectées avec les deux instruments.

La carte synthétique ainsi obtenue présente une tendance globale, liée principalement à un fort gradient de salinité orienté du nord-est vers le sud-ouest, c'est-à-dire vers la lagune (figure 2a). Dans le but de mettre en évidence les fortes variations locales de conductivité, la médiane locale a été calculée sur une fenêtre de $41 \mathrm{~m}$ de côté (figure $2 \mathrm{~b}$ ) puis retirée (figure 3).

La gamme de variations des mesures de conductivité électrique apparente est particulièrement large : les valeurs sont comprises entre 20 et près de $1000 \mathrm{mS} / \mathrm{m}$. Le retrait de la tendance générale souligne beaucoup d'anomalies dans la gamme $\pm 200 \mathrm{mS} / \mathrm{m}$. Seules celles semblant présenter un intérêt archéologique sont mentionnées sur la figure 3. Les anomalies dont l'origine récente a pu être vérifiée, telles celles dues aux rizières qui occupaient une partie du Castélou au milieu du $\mathrm{Xx}^{\mathrm{e}}$ siècle, ne sont donc pas évoquées.

L'anomalie conductrice A, d'une longueur de 250 à $300 \mathrm{~m}$ et d'une largeur de plusieurs dizaines de mètres, possède des limites rectilignes. Elle pourrait correspondre à un tronçon d'un paléochenal comblé dans sa partie supérieure par des sédiments fins plus conducteurs que ses berges. Au nord, une autre bande conductrice $B$ semble être le prolongement de A.
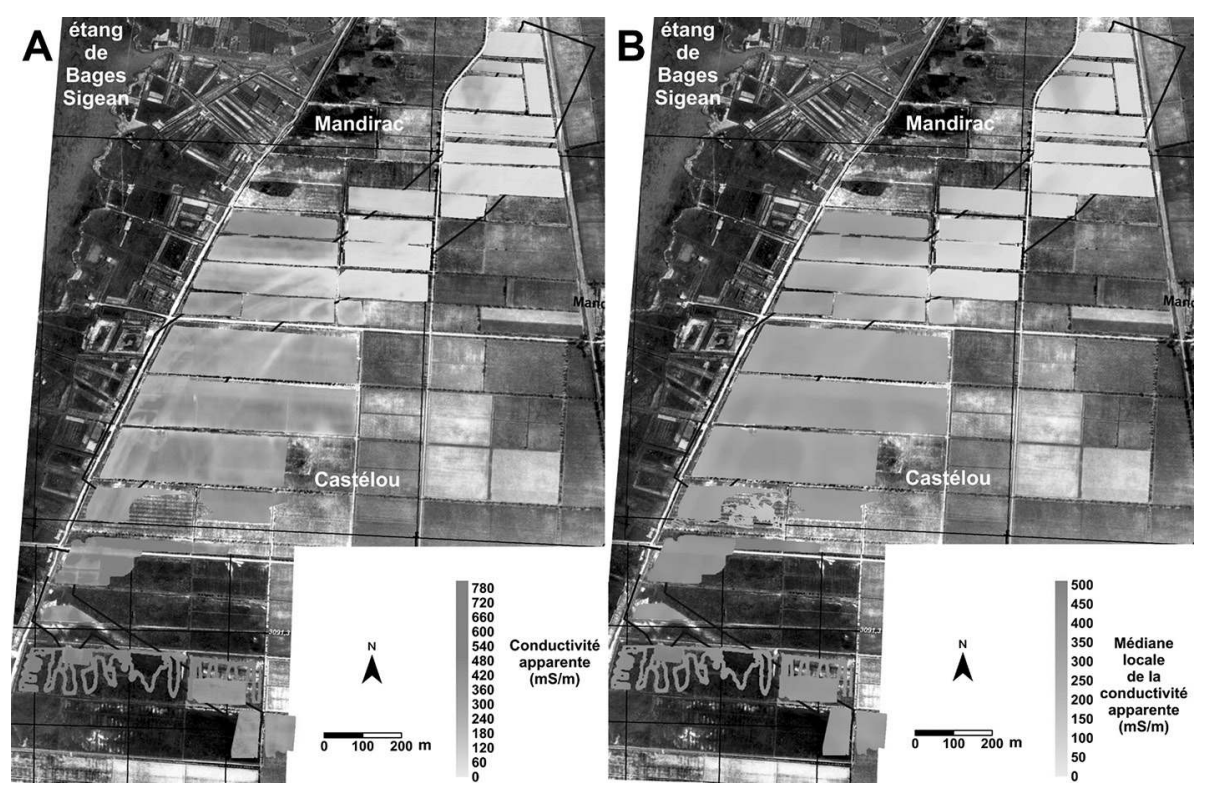

Figure 2 : (Voir planche couleur V) Carte de conductivité électrique apparente. A) Données brutes. B) Médiane locale Figure 2: (See colour plate V) Apparent electrical conductivity map. A) Raw data. B) Local median.

ArCheoSCIEnCES, revue d'archéométrie, 40, 2016, p. 47-63 


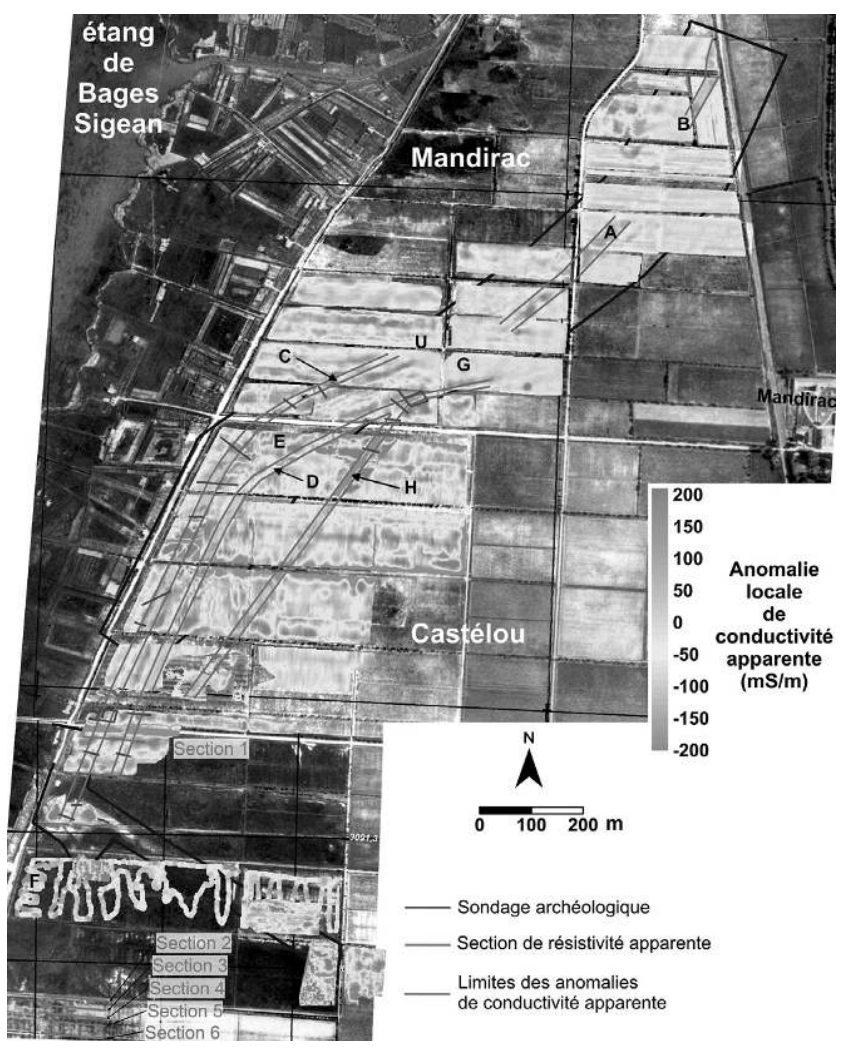

Figure 3 : (Voir planche couleur V) Conductivité électrique apparente : carte des écarts à la médiane locale.

Figure 3: (See colour plate V) Apparent electrical conductivity: map of local median difference.

Au sud-ouest de B, les anomalies sont organisées en longues bandes étroites orientées sud-ouest/nord-est, alternativement conductrices ou résistantes. Les anomalies résistantes $\mathrm{C}$ et $\mathrm{D}$, larges de plus de $15 \mathrm{~m}$ chacune, sont plus particulièrement remarquables. Elles encadrent une surface $\mathrm{E}$ elle-même constituée d'une alternance d'étroites bandes résistantes ou conductrices parallèles entre elles. Au sud, les anomalies $\mathrm{C}$ et $\mathrm{D}$ prennent une direction presque nord/sud. Ces données confirment et précisent les informations relevées sur les photographies aériennes par M. Guy (Falguéra et al., 2000) et appuient les observations faites par H. Rouzaud (Rouzaud, 1914) qui décrivait un probable quai. À l'extrême sud de la zone cartographiée (F), alors que la conductivité est particulièrement élevée $(>500 \mathrm{mS} / \mathrm{m}$, figure $2 \mathrm{a})$, les anomalies $\mathrm{C}$ et $\mathrm{D}$ ne sont plus visibles. À leur extrémité nord $(\mathrm{G})$, les valeurs sont plus faibles (100 à $200 \mathrm{mS} / \mathrm{m}$ ) mais très peu contrastées : les anomalies $C$ et $D$ ne sont plus visibles là non plus. À l'est de l'anomalie $\mathrm{D}$, l'anomalie $\mathrm{H}$ se présente comme une bande résistante d'une dizaine de mètres de large, parfaitement rectiligne sur environ $700 \mathrm{~m}$ de long.
Elle rejoint avec certitude l'anomalie D au nord, alors qu'au sud, sa jonction éventuelle avec cette même structure n'a pas été mise en évidence.

\section{Identification des anomalies de conductivité par la fouille (2008-2013)}

Sur la base de la carte de conductivité électrique, et alors qu'environ 33 ha avaient déjà été prospectés, des sondages archéologiques dirigés par $\mathrm{C}$. Sanchez furent entrepris dès 2008. La localisation et les dimensions de ces investigations purent être choisies très précisément grâce aux données géophysiques. Ce fut d'abord l'anomalie $\mathrm{C}$ qui fut identifiée au travers de six sondages au Castélou mettant en évidence un empierrement large d'environ $12,5 \mathrm{~m}$ vers le nord, s'élargissant vers le sud pour atteindre plus de $17 \mathrm{~m}$ (Cavero et al., 2010). Il présentait deux états successifs séparés par une couche de limons d'une vingtaine de centimètres d'épaisseur, probablement déposée lors d'une crue très importante. En 2009, plusieurs nouvelles tranchées ouvertes dans le secteur de Mandirac ont confirmé l'importance de cet empierrement fondé à la fin du $\mathrm{I}^{\text {er }}$ siècle sur des niveaux instables dans une zone extrêmement marécageuse. Cette structure qui apparait alors comme une voie s'adapte aux milieux qu'elle traverse et assure sa stabilité par différents aménagements. Par endroits le lit de matériaux résistants repose sur une couche d'amphores (figure 4A); ailleurs, il a pour soubassement un radier de pieux battus (figure 4B). Il se compose en grande partie de matériaux de démolition (moellons, pierres de taille sculptées provenant vraisemblablement de bâtiments publics, tuiles et enduits peints portant des traces de feu peut-être issus de domus incendiées, céramiques, etc.) et présente des recharges successives. Son épaisseur est de l'ordre d'1,2 m. En 2010, les vérifications de l'anomalie $\mathrm{C}$ se sont poursuivies vers le sud, au Castélou, mettant en évidence l'aménagement de ses limites, à l'ouest par des pieux maintenant des planches sur lesquelles s'appuie un amas de moellons et, à l'est, par un alignement de blocs non équarris $(0,4 \times 0,6 \mathrm{~m})$. Deux tranchées ont également été ouvertes en travers de l'anomalie $\mathrm{D}$, révélant un autre empierrement massif. Un premier sondage fut aussi réalisé sur l'anomalie $\mathrm{H}$, montrant une structure plus légère que les deux précédentes mais tout de même constituée d'un alignement de blocs calcaire. Un second, effectué en 2011 à hauteur de la jonction avec l'anomalie $\mathrm{D}$, a confirmé ce résultat attribuant à cette structure une fonction de briselame (figure 5). Cette même année, huit carottages furent réalisés au carottier à percussion selon un transect perpendiculaire à $\mathrm{C}$ et à $\mathrm{D}$ (proche de l'emplacement de la section 1, figure 3). Ces investigations ont confirmé la présence 


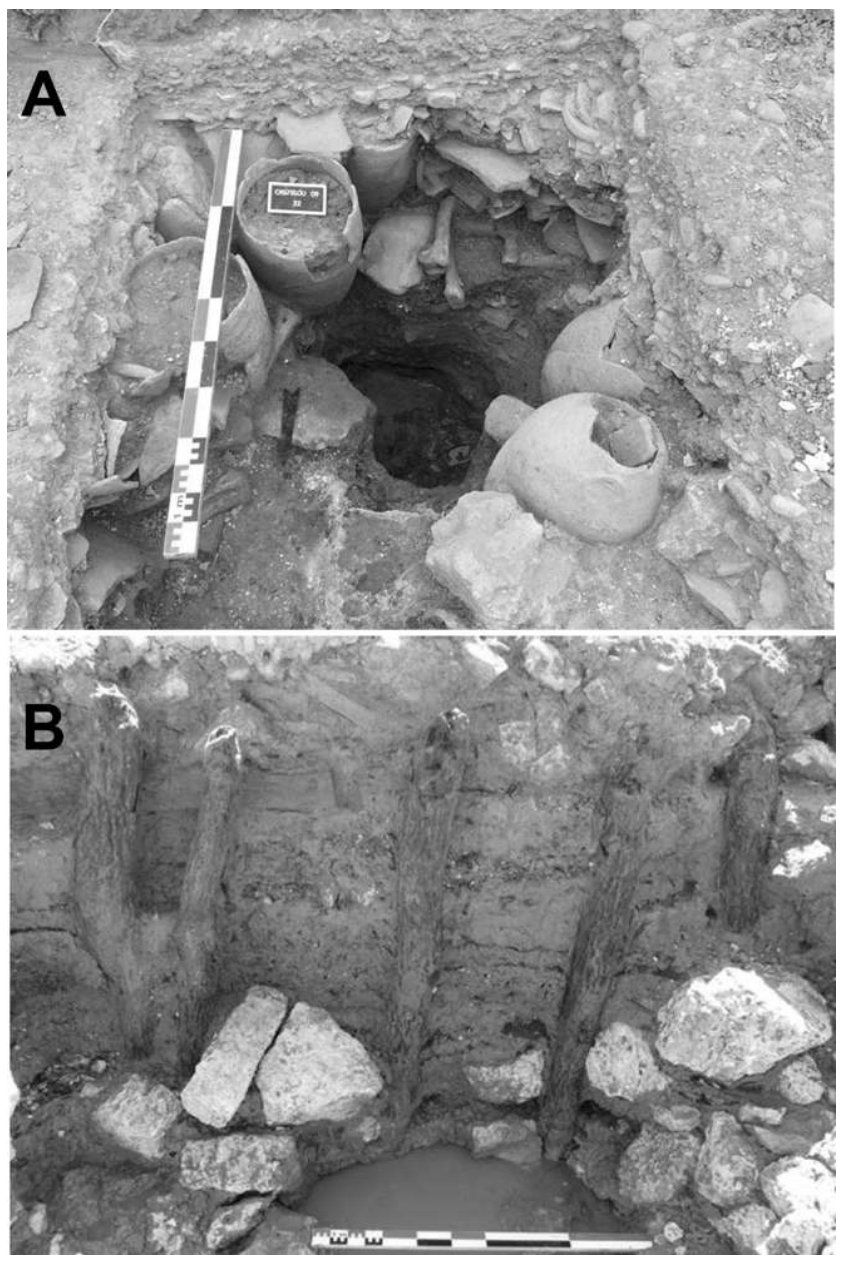

Figure 4 : Mandirac. L'empierrement ouest repose au nord sur des amphores (A) et plus au sud sur des pieux (B). Clichés pris par C. Sanchez.

Figure 4: Mandirac. The west metalling lies north of amphorae (A) and further south on piles (B). Photos taken by $C$. Sanchez.

d'un chenal large de 45 à $50 \mathrm{~m}$ et profond d'environ 3,5 m, bordé par les deux empierrements (figure 6, Sanchez et al., 2014). Il correspond à l'anomalie conductrice E. La poursuite des fouilles jusqu'en 2013 a montré que ces structures présentent d'importants aménagements de berges. Un bâtiment, probablement un entrepôt, a même été découvert au centre de l'empierrement ouest qui présente, à cette endroit, une largeur de près de $25 \mathrm{~m}$.

\section{Sections de résistivité électrique (2015)}

La cartographie de conductivité électrique par méthode Slingram s'est avérée inefficace sur la partie la plus au sud de la zone prospectée. Ceci est probablement lié au fait que la conductivité apparente y est très élevée $(>500 \mathrm{mS} / \mathrm{m})$ et que les structures à l'origine des anomalies résistantes $\mathrm{C}$ et $\mathrm{D}$ sont de plus en plus profondes. En effet les fouilles ont montré qu'elles apparaissent au nord à $0,5 \mathrm{~m}$ sous la surface alors que dans les tranchées les plus au sud elles sont situées à plus d'1 $\mathrm{m}$ de profondeur (Sanchez et al., sous presse). Elles ont donc été recherchées en enfonçant des piques métalliques dans le sol. La terminaison sud des structures a ainsi pu être localisée en 2011 et la méthode a montré que l'empierrement oriental mesurait $55 \mathrm{~m}$ de long de plus que l'empierrement occidental. Le terrain, particulièrement humide et argileux à cet endroit, n'a pas permis de mener à bien les sondages prévus l'année suivante pour étudier ces aménagements situés de part et d'autre de l'embouchure du chenal. Pour palier partiellement à cette impossibilité de relever la géométrie des structures, cinq pseudosections de résistivité apparente ont été acquises en 2015. Afin de faciliter l'interprétation de ces données, une pseudosection a tout d'abord été enregistrée parallèlement et à une vingtaine de mètres d'une bande fouillée ayant aussi fait l'objet d'une dizaine de carottages profonds de 7 à $10 \mathrm{~m}$ (Sanchez et al., 2014).

La section obtenue permet de documenter la structure verticale des anomalies $\mathrm{C}, \mathrm{E}$ et $\mathrm{D}$. Compte-tenu de la forte salinité du milieu, les valeurs de résistivité électrique sont inférieures à $1 \Omega$.m et les contrastes de résistivité sont de l'ordre de $0,1 \Omega . \mathrm{m}$ (figure 7 ). Un bon rapport signal/bruit de la mesure a pu être préservé dans un tel contexte conducteur grâce à l'injection d'un courant électrique intense (1 A). La section apparait cohérente avec les résultats de la prospection électromagnétique. En effet, elle recoupe deux zones résistantes localisées à la verticale des anomalies $\mathrm{C}$ et $\mathrm{D}$. L'anomalie $\mathrm{C}$ apparait en coupe comme une structure résistante de 1,5 à $2 \mathrm{~m}$ d'épaisseur et d'une largeur totale d'environ $25 \mathrm{~m}$. Cet aménagement en pierres plonge vers l'est en suivant une pente à $45^{\circ}$ jusqu'à une profondeur d'environ $5 \mathrm{~m}$. Une anomalie comparable (D), presque symétrique, est située environ $45 \mathrm{~m}$ plus à l'est. Son épaisseur est comprise entre 1 et $1,5 \mathrm{~m}$ et sa largeur est de l'ordre de $18 \mathrm{~m}$. Le plongement vers l'ouest est plus fort. Le chenal (E) apparait comblé par un matériau très conducteur $(\mathrm{J})$ au sein duquel se trouve une masse plus résistante $(\mathrm{K})$ d'une épaisseur de plusieurs mètres. Conformément aux données de carottage, elle correspond à une lentille de sable présente à une profondeur d'environ 3,5 m et recouverte de sédiments plus fins (Sanchez et al., 2014). La profondeur de cette entité qui marque le fond du chenal est suffisante pour que la rivière ait été navigable dans l'Antiquité. Un matériau majoritairement sableux est également présent sous une partie de l'anomalie D (L), mais aussi, et en plus grande quantité, sous l'anomalie $\mathrm{C}(\mathrm{M})$. Ceci laisse à penser que les structures $\mathrm{C}$ et $\mathrm{D}$ s'appuient sur des bancs de sable préexistants. À l'est 
Figure 5 : Mandirac. Vue générale des aménagements de la rive gauche. Cliché pris vers le sud-ouest en 2013 par C. Durand, CNRS/ CCJ. Les anomalies phytologiques du chenal et des structures de la rive droite sont également visibles. En arrière-plan, les étangs de Bages/Sigean.

Figure 5: Mandirac. Overview of facilities on the left bank. Photo taken to the southwest in 2013 by C. Durand, CNRS/CCJ. The phytological anomalies of the channel and the right bank structures are also visible. In the background, the Bages / Sigean pond.
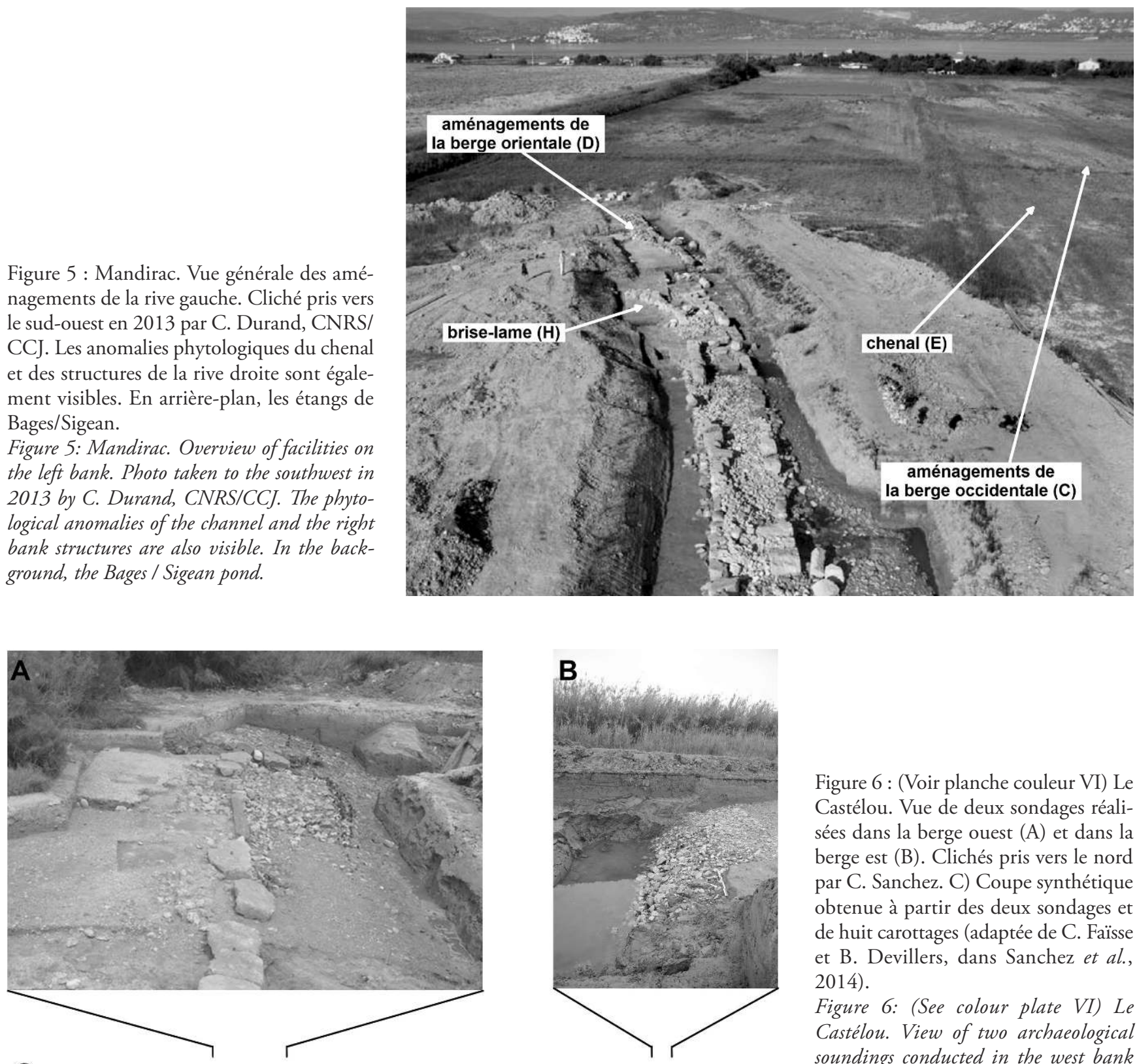

Figure 6 : (Voir planche couleur VI) Le Castélou. Vue de deux sondages réalisées dans la berge ouest (A) et dans la berge est (B). Clichés pris vers le nord par C. Sanchez. C) Coupe synthétique obtenue à partir des deux sondages et de huit carottages (adaptée de C. Faïsse et B. Devillers, dans Sanchez et al., 2014).

Figure 6: (See colour plate VI) Le Castélou. View of two archaeological soundings conducted in the west bank (A) and in the east bank (B). Photos

\section{C}

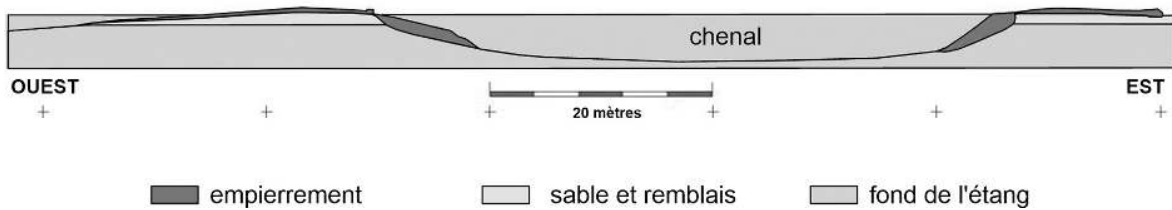

taken to the north by $C$. Sanchez. C) Synthetic section obtained from the two soundings and eight cores (adapted from C. Faïse and B. Devillers, in Sanchez et al., 2014).

de l'anomalie D, le matériau est par contre beaucoup plus argileux $(\mathrm{N})$. Ceci est confirmé par les observations sédimentologiques. L'ensemble de ces formations surmontent une entité résistante $(\mathrm{O})$ dont le toit se situe à une profondeur d'environ $10 \mathrm{~m}$.
On retrouve ces mêmes entités dans la plupart des cinq sections réalisées quelques centaines de mètres plus au sud. La structure occidentale (C) est visible sur les sections 2 et 3 où elle présente une largeur respectivement de 25 et de $20 \mathrm{~m}$. La structure orientale (D) est également présente sur 

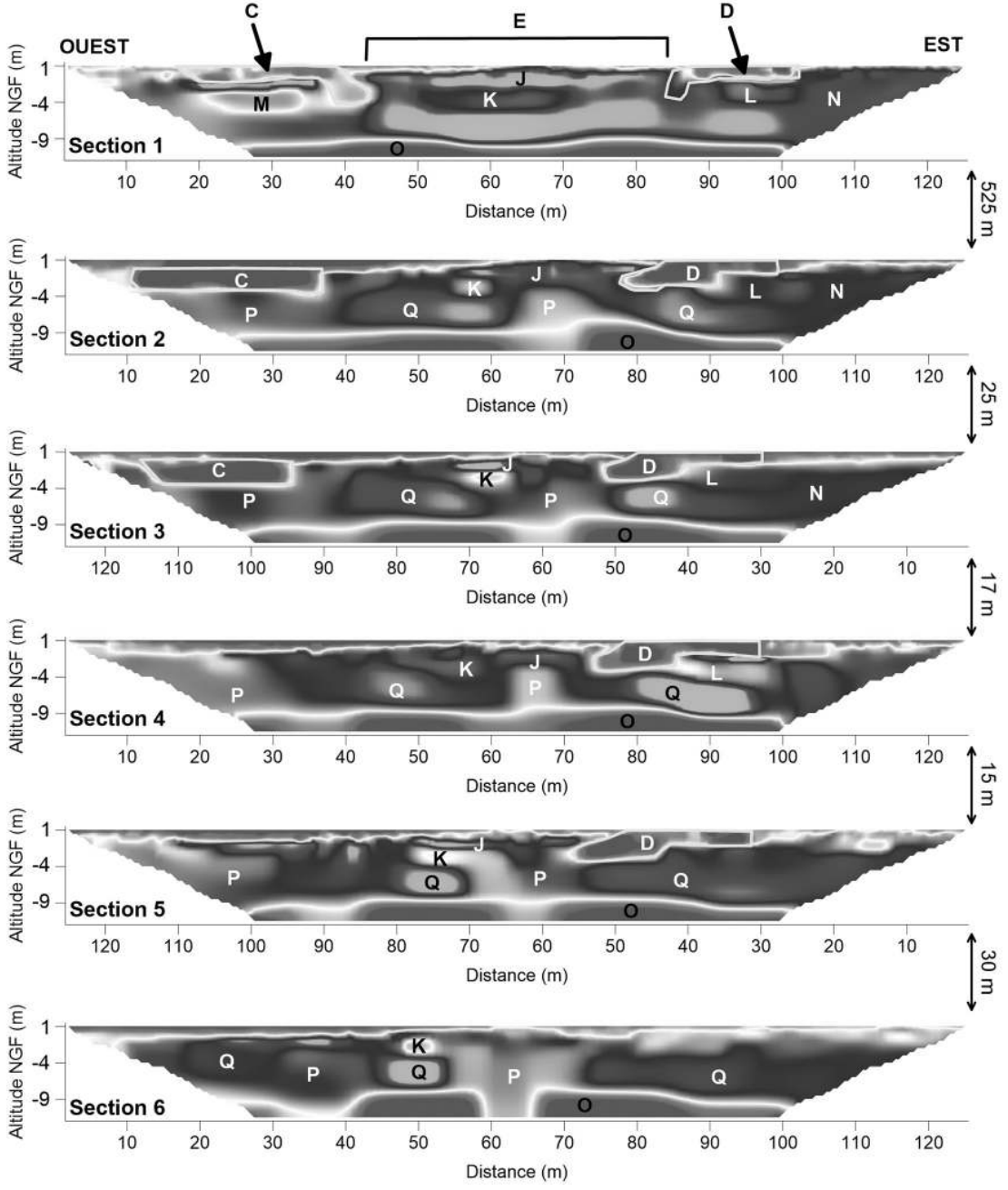

berges antiques

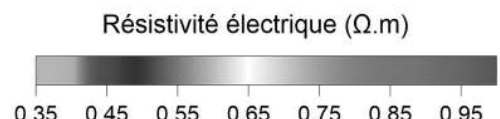

Figure 7 : (Voir planche couleur VI) Le Castélou. Sections de résistivité électrique réalisées à l'extrémité sud du site. Après cinq itérations, l'erreur absolue est de l'ordre de $2 \%(1,34$ à 2,60\%) en utilisant une inversion avec la méthode de contrainte robuste (L1-norm) qui minimise la somme des valeurs absolues des différences entre les résistivités apparentes calculées et mesurées. Figure 7: (See colour plate VI) Le Castélou. Electrical resistivity tomography performed at the southern end of the site. After five iterations, the absolute error is about 2\% (1.34 to $2.60 \%)$ using an inversion with the robust enforcement method (L1 -norm) which minimizes the sum of absolute values of differences between the calculated and measured apparent resistivities. les sections 4 et 5, conformément aux données de piquetage qui indiquaient un décalage de $55 \mathrm{~m}$. Celui-ci a probablement été créé pour faciliter l'entrée dans l'embouchure malgré la dérive littorale, à l'image de l'aménagement de l'embouchure actuel de l'Aude. La largeur de l'empierrement oriental est de l'ordre de $18 \mathrm{~m}$ dans les sections 2 et 3 . Elle croit pour attendre près de $25 \mathrm{~m}$ dans la section 5 . La limite occidentale de la structure D montre un faible pendage vers l'ouest correspondant à une pente de la berge vers le fleuve. La section 6 est située au-delà de l'emprise des berges en pierre. Les sections 2 à 6 présentent beaucoup d'anomalies correspondant à celles identifiées ci-dessus. Entre $-3,5$ et -9 m NGF se succèdent un ensemble de formations tantôt plus résistantes $(\mathrm{P})$ et tantôt plus conductrices $(\mathrm{Q})$, dont la conductivité dépend vraisemblablement de la balance entre teneur en « argiles" et teneur en "sable ».

\section{Cartographie magnétique (2010-2013)}

En 2010, des prospections magnétiques ont été mise en œuvre pour la première fois sur le site afin de tenter d'imager les structures anthropiques sur des zones où la cartographie électromagnétique Slingram fournissait des résultats peu probants. C'était le cas dans le secteur de Mandirac, au niveau de l'anomalie $\mathrm{G}$ et plus au nord. Ces tests sur 3 ha furent particulièrement fructueux et donc suivis de nouvelles prospections en 2012 et 2013 . La surface totale prospectée au magnétomètre est de l'ordre de 21 ha. La chaussée occi- 


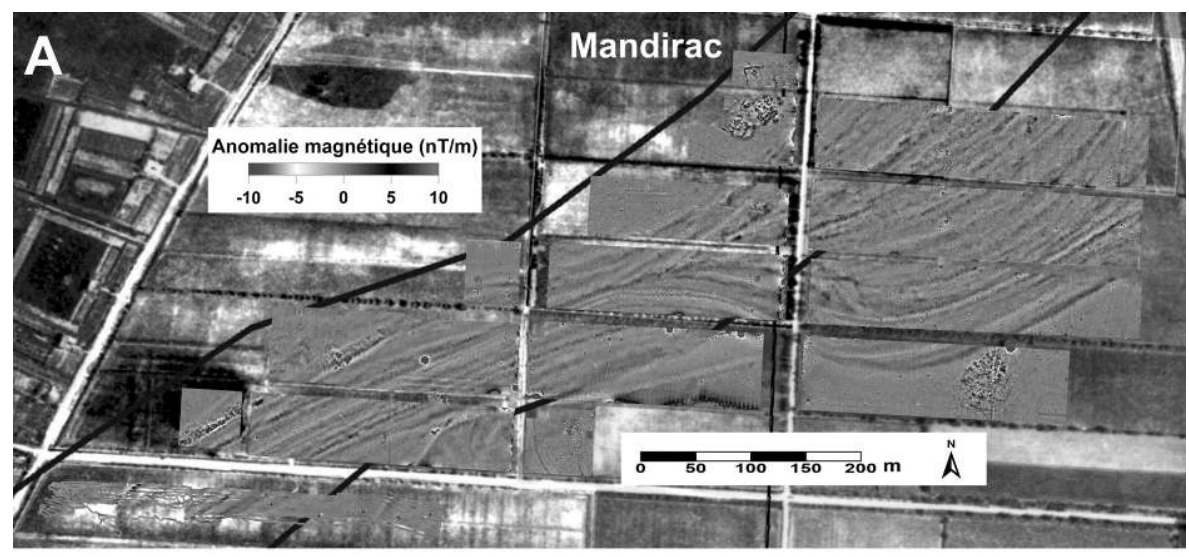

Figure 8 : (Voir planche couleur VII) Mandirac. A) Carte du gradient de la composante verticale du champ magnétique. B) Avec les interprétations. Figure 8: (See colour plate VII) Mandirac. A) Map of vertical gradient component of the magnetic field. B) With interpretations.

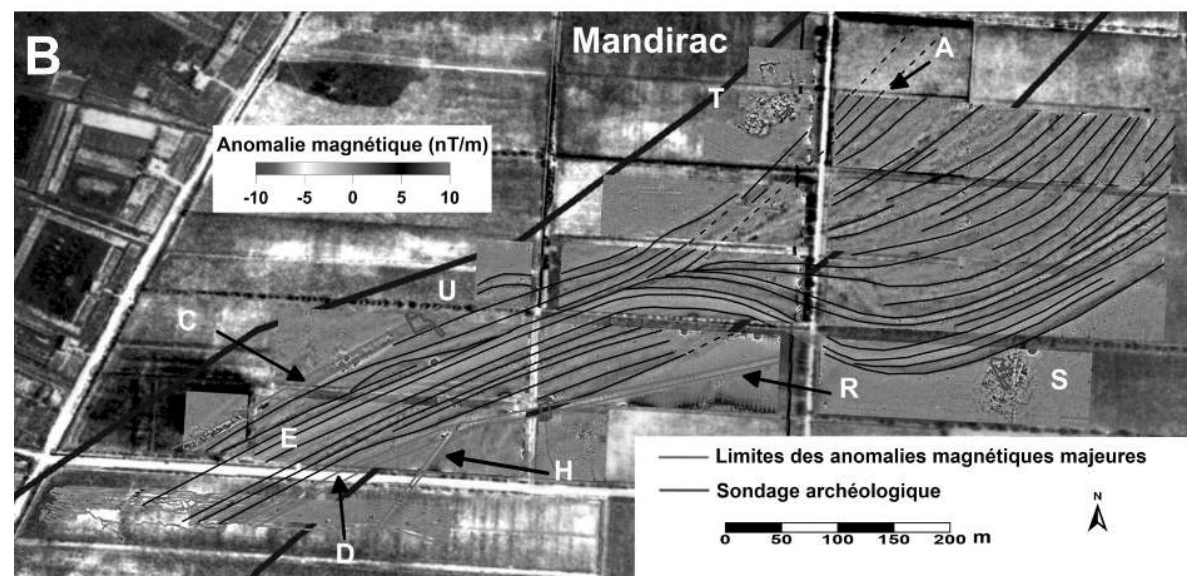

dentale (C) apparait comme une bande où le signal magnétique est modéré mais particulièrement bruité (figure 8). Au nord-ouest de cette entité, la texture de l'image est très différente : le signal est constant, sans anomalie notable. Au sud-est par contre se succèdent des anomalies linéaires subparallèles. Presque la totalité de la surface prospectée présente une alternance d'anomalies magnétiques linéaires positives et négatives (traits noirs). Celles-ci sont pour la plupart orientées sud-ouest/nord-est, c'est-à-dire parallèlement à l'anomalie C. Toutefois, au centre de la zone prospectée, une partie des anomalies est affectée d'un double changement de direction puis d'un retour à une orientation proche de leur orientation initiale. Ces anomalies très caractéristiques ne laissent aucun doute sur leur origine. Elles correspondent à l'enregistrement des divagations d'un important cours d'eau. L'alternance d'anomalies magnétiques linéaires positives et négatives peut simplement s'expliquer par des variations latérales de granulométrie : les fractions fines contiennent généralement plus de minéraux magnétiques que les fractions plus grossières. Le relevé des principales anomalies permet de repérer le lit mineur le plus récent de cette voie d'eau (rose clair). C'est en effet celui qui recoupe les autres. Bien qu'il soit très délicat d'identifier une chronologie relative dans ce type d'environnement particulièrement plat facilitant par conséquent la mobilité du cours d'eau, il semblerait que l'anomalie A puisse correspondre au plus ancien lit visible ici, alors relativement rectiligne. Au nord-est, la voie d'eau s'est peu à peu décalée vers l'est jusqu'à former un méandre large de près de $300 \mathrm{~m}$. La texture de l'image dans les zones non traversées par le cours d'eau est très lisse. Au sud-ouest, le lit est resté très rectiligne, divaguant dans un espace plus restreint, large de moins de $100 \mathrm{~m}$. Dans les parcelles les plus au sud, les deux chaussées semblent totalement contraindre l'écoulement de l'eau. Ces observations renforcent l'hypothèse d'un fleuve canalisé entre les deux chaussées.

Les prospections magnétiques ont également permis de mettre en évidence une anomalie linéaire négative de près de $200 \mathrm{~m}$ de long (R). Elle a été attribuée à une structure large de quelques mètres, donc plus étroite que les deux berges, et constituée de pierres non magnétiques. Elle est reliée à la berge $\mathrm{D}$ à proximité de la jonction entre $\mathrm{D}$ et $\mathrm{H}$. À l'est, cette structure s'interrompt précisément à l'endroit où le méandre est le plus large. Il convient donc de se demander si le méandre a ici dépassé le prolongement de l'ouvrage parce que celui-là n'existait pas, ou bien si c'est le fleuve qui a détruit la construction. 
Une zone située à quelques mètres de l'espace de divagation du fleuve attire l'attention (S). Elle correspond à une grande quantité d'anomalies magnétiques dipolaires de très petites dimensions. L'ensemble, qui apparait organisé spatialement, forme approximativement un ovale positionné à cheval sur une ancienne limite de parcelle.

Plus au nord-est, un groupe d'anomalies situé en dehors de l'espace de divagation du cours d'eau a été repéré (T). Une forte concentration de dipôles créant un signal magnétique très bruité s'explique par la présence de nombreux fragments de matériaux magnétiques à la surface du sol (fragments de terre cuite et de roche volcanique). À proximité immédiate, plusieurs anomalies linéaires positives se recoupent à angles droits. Compte tenu du signal et de la nature des matériaux présents sur le site, ces anomalies pourraient correspondre soit à des fossés, soit à des murs récupérés dont seules subsisteraient les tranchées de fondation.

\section{Identification de trois anomalies magnétiques par la fouille (2011-2013)}

Une fouille a été réalisée en 2011 et 2012 sur l'étroite anomalie linéaire négative $\mathrm{R}$ de près de $200 \mathrm{~m}$ de long (figure 8 ). Elle a mis au jour un aménagement constitué d'une digue et de caissons. Cette digue empierrée est encadrée au nord par un alignement de pieux espacés d'environ $1 \mathrm{~m}$ et au sud par une palée de pieux accolés rigidifiés par des madriers placés de part et d'autre (figure 9). L'empierrement de la digue est composé de moellons, de blocs équarris et d'éléments architecturaux (colonnes, chapiteaux, architraves...). Les caissons, situés au sud contre la palée en bois, semblent construits après la digue, avec des matériaux de récupération. Ils sont constitués de pieux et de planches de typologies variées formant des compartiments de $10,5 \mathrm{~m}$ de long remplis de blocs, de sables et de graviers. Ils ont pour fonction de protéger la digue des " coups de mer " provenant de la

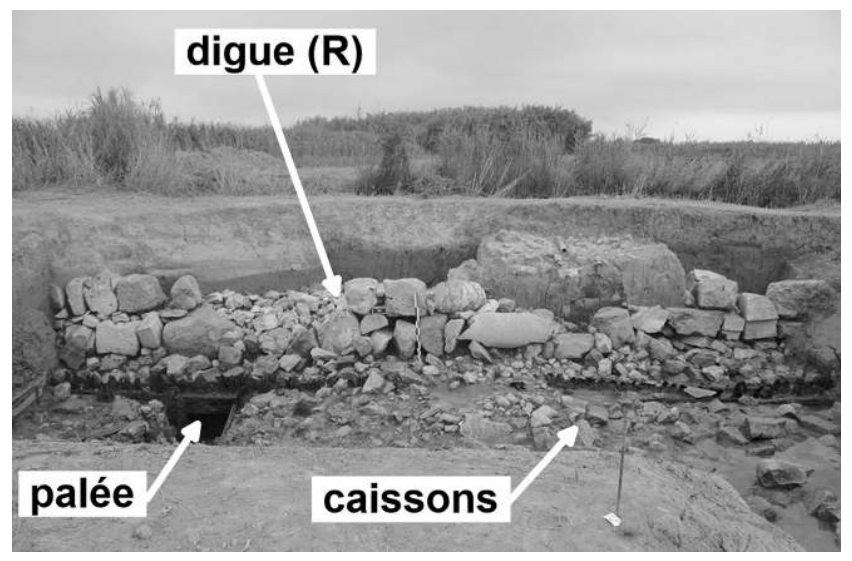

lagune au sud. Comme le montre très bien la carte d'anomalie magnétique et comme a pu l'attester la fouille, la digue est située dans la continuité de la chaussée orientale. Il s'agit d'un seul et même aménagement entre le fleuve au nord et la lagune au sud.

L'anomalie $S$ formant approximativement un ovale de $1600 \mathrm{~m}^{2}$ a fait l'objet d'un sondage de vérification en 2012 . Cette zone est située à quelques mètres de l'espace de divagation du fleuve. Aucune structure linéaire n'était visible sur la cartographie magnétique, l'ensemble étant composé d'une grande quantité de dipôles magnétiques de très petite dimension. Compte tenu de leur position, ces dernières pouvaient être liées à une occupation contemporaine de l'époque où le fleuve était situé à proximité. Afin de vérifier la nature de cette anomalie une ouverture de $130 \mathrm{~m}^{2}$ a été réalisée. Le caractère bruité de l'image magnétique a rapidement été expliqué par un nappage compact d'amphores et de coquillages qui recouvre des niveaux d'occupation associés à des limites bâties. L'occupation, datable du $\mathrm{I}^{\mathrm{er}} \mathrm{s}$. av. n. è. est installée sur un îlot sableux au sein d'un milieu calme révélé par des dépôts argilo-limoneux jaunâtres et fins déposés contre le talus formé par les vestiges. Cet îlot témoigne d'une installation spécifique pour l'exploitation des produits de la mer antérieure aux aménagements de l'embouchure du fleuve, mais aussi d'une topographie complexe avec des zones émergées dans la lagune.

Une fouille a été réalisée en 2013 à proximité de l'extrémité nord de l'anomalie $\mathrm{C}$, à l'endroit où le fleuve est le plus large et où son cours est dévié pour entrer dans le chenal. Trois phases ont pu être mises en évidence. La première correspond à la mise en place d'une voie d'environ $12 \mathrm{~m}$ de large, construite directement sur le fond de la lagune. Elle est composée d'un lit de galets provenant probablement du plateau de Quatourze distant de quelques kilomètres, bordé à l'ouest par amas de gros blocs calcaire et à l'est par une palée. Ce premier état est scellé par des sédiments de crue. Durant la seconde phase on constate un élargissement et un rehaussement du niveau de voie qui mesure alors $21 \mathrm{~m}$ de large. Sur l'espace de circulation cela se matérialise par un grand apport de galets. Sur la partie est de la chaussée, ce rehaussement est identifiable par un apport de sédiments, mélange semblant provenir du fleuve et être le résultat d'un dragage. L'ensemble est recouvert par plusieurs recharges qui uniformisent l'espace de circulation. Ces derniers niveaux

Figure 9 : Mandirac. Vue de la digue placée en rive gauche du chenal (cliché pris vers le nord par C. Sanchez).

Figure 9: Mandirac. View of the dam located along on the left bank of the channel (photo taken north by C. Sanchez). 
alternent avec des dépôts de crues témoins du débordement du fleuve hors de l'espace canalisé. L'épaisseur de la structure passe alors de 0,44 $\mathrm{m}$ (état 1) à un total de 1,30 $\mathrm{m}$ (état 2) sur la partie centrale L'abandon de la structure se traduit par une couche de limon pouvant atteindre par endroits $0,9 \mathrm{~m}$ d'épaisseur. Cette fouille n'a pas repérée de lieu de déchargement contrairement à la zone située en face, sur l'autre berge, ou beaucoup plus au sud, sur cette même berge. Dans ces deux cas, des aménagements particuliers et beaucoup de mobilier, témoins d'une activité intense, avaient été retrouvés. La partie nord de la berge ouest pourrait avoir été utilisée comme une voie de halage.

\section{Pénétrométrie dynamique (2011-2013)}

Plusieurs campagnes de prospection au pénétromètre dynamique ont été réalisées afin de repérer le prolongement de la berge ouest vers le nord, au-delà de la zone fouillée décrite précédemment. 34 essais pénétrométriques ont été effectués dans le voisinage de la zone $U$ où ni les prospections électromagnétiques (figure 7), ni les prospections magnétiques (figure 8), n'avaient été en mesure de détecter cet aménagement. Parmi les 34 essais, 20 ont été stoppés lorsqu'ils ont atteint un niveau impossible à traverser. $\mathrm{La}$ profondeur de ce niveau est comprise entre 0,57 et 1,65 m. Les 14 autres essais ont été poursuivis jusqu'à une profondeur d'au moins $3 \mathrm{~m}$ voire le plus souvent jusqu'à 4,4 m. Les 34 essais permettent d'établir une carte synthétique mettant clairement en évidence le tracé de la berge et surtout son interruption brutale au nord (figure 10). En effet, en limite nord-est, l'empierrement présent à moins d' $1 \mathrm{~m}$ de la surface en cinq points plonge fortement (plus d'1,5 m en deux points) avant de disparaitre à quelques mètres de là. Compte tenu de la forme irrégulière de cette interruption, il est raisonnable d'émettre l'hypothèse que cette structure a été détruite partiellement et qu'il ne s'agit donc pas de l'extrémité originelle de cet aménagement. Un autre indice évoque une érosion de la structure : si l'on prolonge en ligne droite le tracé de la berge repérée en prospection magnétique, un essai se trouve nettement à l'intérieur de l'empierrement. Pourtant, il a pu sans problème être poursuivi jusqu'à $4.5 \mathrm{~m}$ sans rencontrer de niveau dur. Une destruction partielle de l'aménagement semble plus probable que son rétrécissement.

\section{DisCusSION}

\section{Sur la nécessité d'adapter la méthodologie}

Le choix d'une méthode de prospection géophysique pour traiter une problématique archéologique dépend avant tout de la nature des vestiges recherchés et du milieu étudié. La détection de larges aménagements en pierres au sein d'une zone humide, qui plus est lorsque celles-ci bordent un paléochenal, est aisée tant les contrastes de résistivitél conductivité électriques sont importants. Deux méthodes de cartographie semblent alors s'imposer : la mesure de la résistivité électrique par injection de courant et la prospection électromagnétique Slingram. La première nécessite de planter des électrodes dans le sol : elle ne pouvait donc être utilisé au Castélou compte-tenu de la végétation parfois très dense et haute de près d' $1 \mathrm{~m}$. D'autre part, la très faible résistivité du sol imposait d'injecter une importante quantité de courant (de l'ordre de $1 \mathrm{~A}$ ) ce qui aurait nécessité le déplacement de lourdes batteries (modèle pour voiture). La prospection électromagnétique Slingram présentait pour sa part
Figure 10 : (Voir planche couleur VII) Mandirac. Carte synthétique regroupant 34 essais pénétrométriques réalisés entre 2011 et 2013. Les limites de la berge ouest (traits noirs) sont proposées à partir des résultats des prospections magnétiques et des essais pénétrométriques. Les pointillés bleus indiquent les zones probables d'arrachement de cette structure en pierres. Figure 10: (See colour plate VII) Mandirac. Synthetic map grouping 34 cone penetration tests performed between 2011 and 2013. Limits of the west bank (black lines) are proposed based on the results of the magnetic survey and cone penetration tests. The blue dotted lines indicate the likely areas of erosion of this stone structure.

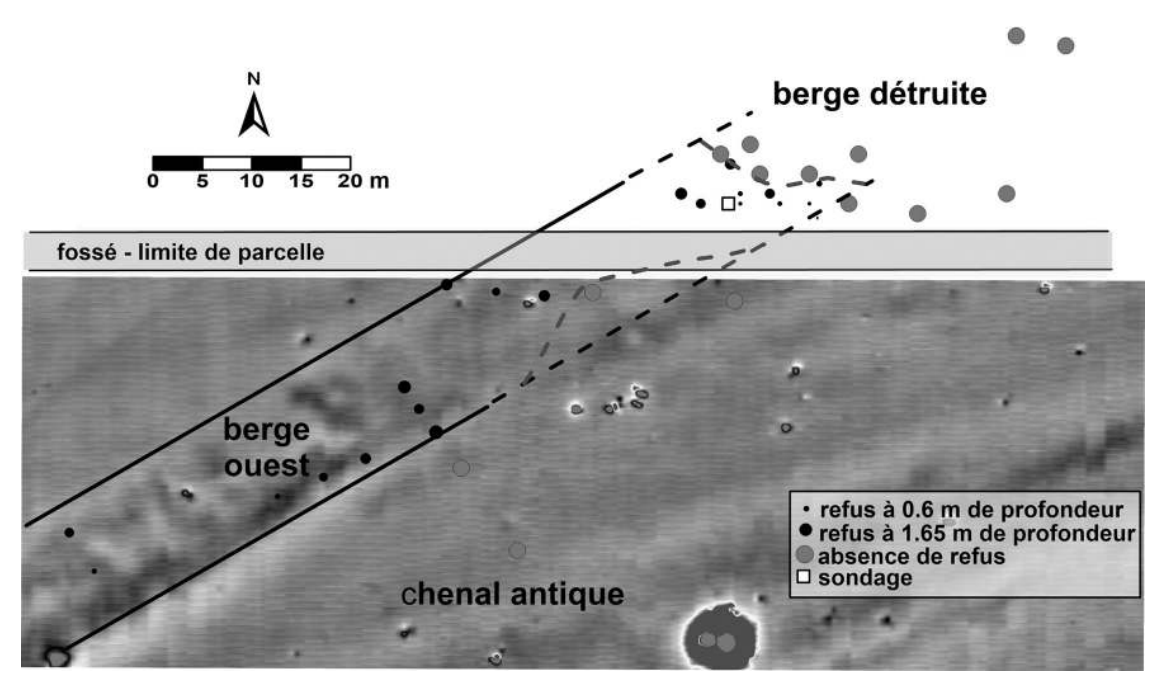


l'intérêt de consommer beaucoup moins d'énergie (quelques piles alcalines suffisent pour plusieurs jours) et de permettre d'évoluer au-dessus de la végétation si nécessaire. Grâce à sa grande facilité de mise en ouvre, la structure majeure du site, une voie d'eau antique bordée de deux structures en pierres larges de 12 à $25 \mathrm{~m}$ et espacées d'au moins $45 \mathrm{~m}$, a pu être mise en évidence sur une distance d'environ 1,2 km. Ce travail correspond à environ 20 jours de prospection.

L'extrémité sud de chacun de ces aménagements n'ayant pu être localisée en raison d'une perte progressive du contraste de conductivité électrique probablement lié à l'approfondissement du toit des structures, il a été nécessaire de changer de méthode d'exploration. Les empierrements ont été suivis jusqu'à leur extrémité, $500 \mathrm{~m}$ plus au sud, par piquetage puis imagés par des sections électriques rapprochées puisque la nature du terrain ne permettait pas d'y engager des fouilles.

Suite à la découverte en 2010 d'un bâtiment reposant sur la berge ouest, il est apparu opportun de mettre en œuvre une technique à plus haute résolution spatiale permettant de détecter de tels vestiges. Au nord, dans le secteur de Mandirac, sur des terrains plats à la végétation rase ou absente, nous avons choisi de mettre en œuvre un protocole de cartographie magnétique capable de fournir les résultats escomptés. Les prospections ont permis de montrer les capacités de détection des berges par cet instrument, précisant largement les données de conductivité, mais n’ont pas mis en évidence de nouveaux bâtiments. Par contre, grâce au gain de résolution spatiale, une digue non détectée par la méthode électromagnétique a été découverte sur une distance d'environ $200 \mathrm{~m}$, dans le prolongement de la berge est. Malgré la pertinence évidente de la méthode magnétique pour détecter les empierrements sur cette zone, aucune structure n'a été découverte dans le prolongement de la berge ouest, au-delà de l'anomalie résistante détectée par le conductivimètre. Ceci a constitué un premier élément pour évoquer l'arrêt de cette structure, confirmé par la suite par une série d'essais au pénétromètre.

\section{Apports mutuels des prospections géophysiques et des fouilles}

Ces recherches constituent un exemple d'une approche interdisciplinaire dans ces milieux spécifiques. Les prospections géophysiques et les fouilles se sont mutuellement enrichies pour la compréhension de ces aménagements complexes. Sur la base de la synthèse des photographies aériennes et des cartes anciennes, les prospections géophysiques ont permis de détecter et de localiser précisément les structures archéologiques majeures qui étaient recherchées. Environ 80 ha ont été cartographiés de manière exhaustive en une trentaine de jours (prospections électromagnétique et magnétique). Le bilan est exceptionnel : quatre anomalies linéaires ont été repérées correspondant aux deux berges empierrées encadrant le chenal $(1,2 \mathrm{~km})$, à la digue prolongeant la berge est $(200 \mathrm{~m})$ et au brise-lame $(700 \mathrm{~m})$. Ces investigations ont été essentielles pour implanter la plupart des sondages archéologiques. Les dimensions de ceux-ci ont pu être restreintes aux seules zones d'intérêt, limitant au maximum l'impact de ces investigations sur le milieu, un facteur déterminant pour pouvoir travailler sur le long terme dans la réserve naturelle du Castélou.

En retour, les sondages ont permis d'identifier précisément la nature, les dimensions et la profondeur des vestiges archéologiques à l'origine des anomalies géophysiques. Ces vérifications étaient essentielles pour mieux comprendre le signal géophysique, ses limites et ses variations. La qualité des interprétations des zones n'ayant pas été fouillées a ainsi pu être améliorée. Les sondages ont également permis de s'affranchir de certains leurres correspondant à des aménagements modernes, tels des fossés de drainage ou des drains en céramique.

Le travail qui a été mené sur l'extrémité sud de l'embouchure aménagée est un bel exemple de complémentarité interdisciplinaire. Face à l'impossibilité des prospections électromagnétiques à détecter les aménagements sur cette zone, il a été dans un premier temps décidé d'y abandonner les recherches géophysiques pour se consacrer à un repérage par piquetage. Ces travaux ont permis d'évaluer la position des structures au travers d'un semis d'une soixantaine de points. Cette opération, certes importante car elle localisait la terminaison sud des structures, ne pouvait être une fin en soi. Elle devait être suivie par une fouille qui s'est vite révélée impossible en raison de la très faible portance du sol qui a nécessité une évacuation en urgence de la pelle mécanique de la parcelle. L'acquisition quelques années plus tard d'un système d'acquisition de sections électriques a apporté une solution pour obtenir malgré tout la géométrie des structures recherchées (largeur, épaisseur, pente des berges) dans ce milieu particulièrement difficile. Cependant, c'est parce que les résultats d'une section réalisée à proximité immédiate d'un sondage ont été confrontés aux données issues de la fouille que l'interprétation de ces prospections a pu être précise et atteindre les objectifs fixés. L'apport mutuel entre fouille et géophysique a été ici particulièrement probant.

\section{Apport mutuels des prospections géophysiques et des investigations géomorphologiques}

Les données issues des prospections géophysiques ont également été largement confrontées aux études géomor- 
phologiques. Les résultats des carottages ont notamment permis de compléter l'étalonnage de la section de résistivité électrique citée précédemment en identifiant précisément la nature sédimentaire des anomalies de conductivité. En retour, la section a fait le lien entre les différents carottages, offrant une vision exhaustive, en coupe, de la géométrie des corps sédimentaires traversés. Les informations recueillies ont ensuite été extrapolées aux cinq autres sections, apportant ainsi des informations géomorphologiques dans une zone n'ayant pas été carottée.

D'autre part, les différents outils de cartographie géophysique ont mis en évidence des structures géologiques de surface utile pour comprendre l'évolution géomorphologique de la zone. Plusieurs tronçons de paléochenaux ont notamment été localisés par la prospection électromagnétique. La recherche de bâtiments et de la continuité nord des empierrements par la prospection magnétique nous a également conduit à mettre évidence un grand nombre d'anomalies linéaires subparallèles correspondant aux corps sédimentaires déposés par le cours d'eau au fil des siècles. Le repérage d'une alternance de sédiments fins ou plus grossiers renseigne sur les divagations du fleuve et permet même d'avancer quelques éléments de chronologie relative. Ces investigations ont été déterminantes pour le choix d'implantation de carottages ou de tranchées destinées à identifier plus précisément les sources naturelles à l'origine de ces anomalies géophysiques.

\section{CONCLUSIONS ET PERSPECTIVES}

Les prospections géophysiques, à l'image de celles menées à Portus (Keay et al., 2005), apparaissent comme un des piliers des recherches archéologiques menées sur le système portuaire antique de Narbonne depuis 2005. Elles ont dû évoluer au fil des campagnes de mesures afin de s'adapter aux contraintes du terrain, à l'évolution des résultats et à l'émergence de nouvelles problématiques. Les remarquables résultats obtenus sont avant tout le fruit d'une étroite collaboration entre archéologues, géomorphologues, géographes et géophysiciens. Les prospections ont repéré les structures majeures du site du Castélou/Mandirac identifié comme étant l'embouchure aménagée d'un bras sud de l'Aude, une artère fluviale qui a changé de cours au fil du temps. Les fouilles ont confirmé la présence de deux jetées parallèles construites en milieu humide sur une longueur de plus d'1,5 km et encadrant le fleuve large d'une cinquantaine de mètres pour une profondeur de $4 \mathrm{~m}$ environ. D'une emprise de $15 \mathrm{~m}$ à $25 \mathrm{~m}$, elles sont aménagées en chaussée dès la fin du i $\mathrm{I}^{\text {er }}$ siècle par l'apport de quantités considérables de matériaux et de milliers de pieux en bois qui viennent renforcer leurs berges. Ces chaussées ont été le siège d'une activité intense. Elles semblent présenter un rôle important dans l'embarquement / débarquement de marchandises ou bien encore pour le halage des navires. D'imposants ouvrages viennent compléter l'aménagement des lieux : une digue et un brise-lame d'une longueur respective de 200 et de $700 \mathrm{~m}$. Nulle part dans le monde romain, des constructions de cette ampleur pour canaliser un fleuve ou pour se protéger des assauts de la mer n'ont été mises au jour. Elles soulignent le besoin de maîtriser un milieu fluctuant afin d'assurer une stabilité et une pérennité de ce lieu d'échanges. Les ouvrages présentent non seulement les stigmates des tempêtes et des crues du fleuve (par exemple l'interruption nette de la berge ouest mise en évidence par une série d'essais au pénétromètre), mais aussi de multiples réfections preuve de la volonté de les maintenir en état malgré l'hostilité du milieu. Le site sera finalement abandonné, probablement à la fin du ve siècle.

À l'issue de cette dizaine d'années de recherches interdisciplinaires, des questions restent en suspens et notamment sur la partie amont de l'Aude, vers Narbonne, qui reste à explorer, Les travaux colossaux qui ont été réalisés au Castélou et à Mandirac dans la seconde moitié du $\mathrm{I}^{\text {er }}$ siècle ne mettent pas en œuvre les techniques habituelles de constructions en zone portuaire, comme l'utilisation de béton hydraulique. Les quais bien construits, ostentatoires, que l'on retrouve ailleurs à cette période, sont ici absents, comme de grands entrepôts. Le Castélou/Mandirac ne semble donc constituer « que " l'extrémité du fleuve canalisé. Il faudra donc remonter vers la ville pour chercher de véritables quais « bien construits » et de grands entrepôts. C'est sans doute là la spécificité du port de Narbonne : sa complexité, son ampleur et son éclatement géographique. Le fleuve reste cependant la colonne vertébrale de ce système et le port urbain est encore à mettre en évidence. C'est l'objectif principal des nouvelles campagnes de prospection géophysique organisées depuis 2015.

\section{Remerciements}

Ces campagnes de prospection ont reçu le soutien financier de la région Languedoc-Roussillon, du ministère de la Culture (DRAC et DRASSM) et du LabEx ARCHIMEDE au titre du programme "Investissement d'Avenir " ANR-11LABX-0032-01. Nous remercions vivement Marion Druez (ULR Valor) qui a largement contribué à l'acquisition et au traitement des données géophysiques, ainsi que les relecteurs qui ont activement participé à l'amélioration de cet article. 


\section{Bibliographie}

Ambert P., 1993. Preuves géologiques de l'insularité du massif de la Clape (Aude) pendant la transgression flandrienne. Comptes rendus de l'Académie des Sciences, 316, p. 237-244.

Ambert P., 2000. Narbonne antique et ses ports, géomorphologie et archéologie, certitudes et hypothèses. Revue archéologique de Narbonnaise, 33, p. 295-307.

BeAmish D., 2011. Low induction number, ground conductivity meters: a correction procedure. Journal of Applied Geophysics, 75 , p. 244-253.

Callegary J.B., Ferré T.P.A., Groom R.W., 2007. Vertical spatial sensitivity and exploration depth of low-induction-number electromagnetic induction instruments. Vadose Zone Journal, 6, p. 158-167.

Camus A., Mathé V., Augereau B., Kernéïs E., Lévêque F., Pouget F., 2011. Intégration de la géophysique et de la télédétection pour la cartographie des sols à haute résolution spatiale : exemple de la reconnaissance de paléochenaux historiques dans les marais charentais. Revue Française de Photogrammétrie et de Télédétection, 193, p. 12-26.

Cannon M.E., McKenzie R.C., Lachapelle G., 1994. Soil salinity mapping with electromagnetic induction and satellitebased navigation methods. Canadian Journal of Soil Science, 74, p. 335-343.

Cavero J., 2010. Paléogéographie des étangs narbonnais d'après les sources cartographiques anciennes. Géocarrefour, 85 : 29-40.

Cavero J., Druez M., Günter-Martin H., Jézégou M.-P., Mathé V., Sanchez C., Storch K., 2010. Les ports antiques de Narbonne: approche méthodologique et premiers résultats. In L. Hugot, L. Tranoy (ed.). Les structures portuaires de l'arc atlantique dans l'Antiquité, bilan et perspectives de recherche. Ausonius Editions, Pessac, p. 121-147.

Delefortrie S, Saey T., Van De Vijver E., De Smedt P., Missiaen T., Demerre I., Van Meirvenne M., 2014. Frequency domain electromagnetic induction survey in the intertidal zone: Limitations of low-induction number and depth of exploration. Journal of Applied Geophysics, 100, p. 14-22.

Falguéra F., Falguéra J.-M., Guy M., Marsal A., 2000. Narbonne: cadre naturel et ports à l'époque romaine. Méditerranée, 94, p. 15-24.

Falguéra J.-M., Bernard H, Jézégou M.-P., 2003. Données d'archéologie sous-marine récentes à Port-la-Nautique : pour une approche du système portuaire narbonnais. In G. Perez Ballester, J. Pascual Berlanga (ed.). Puertos fluviales antiguos : ciudad, desarrollo e infraestructuras, IV Jornadas de Arqueologia subacuatica. Facultat de Geographia y Historiad, Valencia, p. 203-212.
Guy M., 1955. Les Ports antiques de Narbonne. Revue d'études ligures, 21, (3-4), p. 213-240.

Johnston M.A., Savage M.J., Moolman J.H., du Plessis H.M., 1997. Evaluation of calibration methods for interpreting soil salinity from electromagnetic induction measurements. Soil Science Society of America Journal, 61, p. 1627-1633.

Keay S., Millet M., Paroli L., Strutt K. (ed.), 2005. Portus: An archaeological survey of the Portus of imperial Rome. Archaeological Monographs of the British School at Rome, London.

Lenoble A., Martinaud M., 2003. Apports du pénétromètre à la connaissance d'un site préhistorique. Le cas de l'abri de Diepklloof, Province du Cap, Afrique du Sud. Revue d'Archéométrie, 27, p. 27-36.

Mankin K.R., Karthikeyan R., 2002. Field assessment of saline seep remediation using electromagnetic induction. Transactions of the American Society of Agricultural Engineers, 45 (1), p. 99-107.

Martinaud M., 1999. Sur l'intégration de résultats géophysiques avec des résultats de sondages mécaniques ponctuels. Revue d'Archéométrie, 23, p. 33-45.

Mathé V., 2010. Apports de la prospection électromagnétique à la question de l'embarcadère de l'agglomération antique de Brion (Saint-Germain-d'Esteuil, Gironde). Aquitania, 26 : 137-146.

Mathé V., Camus A., Martinaud M., Barraud D, Pichonneau J.-F., Tassaux F., 2012. Prospections géophysiques multiméthodes du site gallo-romain de Brion (Gironde, France) : une agglomération secondaire en zone humide. ArchéoSciences, 36, p. 173-189.

McNeIll J.D., 1980a. Electromagnetic terrain conductivity of soils and rocks. Geonics Limited, Mississauga, Canada.

McNeIll J.D., 1980b. Electromagnetic terrain conductivity measurement at low induction numbers. Geonics Limited, Mississauga, Canada.

Morris E.R., 2009. Height-above-ground effects on penetration depth and response of electromagnetic induction soil conductivity meters. Computers and Electronics in Agriculture, 68, p. $150-156$.

Reynolds J.M., 1997. An Introduction to Applied and Environmental Geophysics. John Wiley \& Sons Ltd, Chichester.

Rouzaud H., 1914. Note sur les ports antiques de Narbonne. Bulletin de la commission archéologique et littéraire de Narbonne, 13, p. 279-299.

Rouzaud H., 1916. Note sur les ports antiques de Narbonne (suite). Bulletin de la commission archéologique et littéraire de Narbonne, 14, p. 167-197.

Sanchez C., Dellong E., Guerre J., Loppe F., Moulis D., 2000. Un établissement littoral antique, l'île Saint-Martin à Gruissan (Aude). Revue d'Archéologie Narbonnaise, 33, p. 309-349. 
SANChez C., JÉzÉgou M.-P. (ed.), 2011. Espaces littoraux et zones portuaires de Narbonne de sa région dans l'Antiquité. Monographies d'Archéologie Méditerranéenne, 28, ADAL, Lattes.

Sanchez C., Faïsse C., Jézégou M.-P., Mathé V., 2014. Le système portuaire de Narbonne antique : approche géoarchéologique. In L. Mercuri, R. González Villaescusa, F. Bertoncello (ed.). Implantations humaines en milieu littoral méditerranéen: facteurs d'installation et processus d'appropriation de l'espace, (Préhistoire, Antiquité, Moyen Âge). Actes des $\mathrm{XXXIV}^{\mathrm{e}}$ rencontres internationales d'archéologie et d'histoire d'Antibes, Éditions APDCA, Antibes, p. 125-136.

Sanchez C., Labussière J., Jézégou M.-P., Mathé V., Cavero J., sous presse. L'embouchure du fleuve antique dans les étangs narbonnais. In C. Sanchez, M.-P. Jézégou (éd.), Les ports dans l'espace Méditerranéen antique. Narbonne et les systèmes portuaires Auvio-lagunaires. Supplément à la Revue Archéologique de Narbonnaise, Université Paul-Valéry, Montpellier.
Solier Y., Guy M., Lavagne G.F., Morrisson C., Chevalier Y., Sabrié M., Sabrié R., Bouscaras A., Depeyrot G., Marichal R., 1981. Les épaves de Gruissan. Archaeonautica, 3, p. 7-264.

Van Der Lelij A., 1983. Use of Electromagnetic Induction Instrument (type EM-38) for Mapping Soil Salinity. Water Resources Commission, Murrumbidgee Division, New South Wales, Australia.

Verdeil P., 1970. Données nouvelles sur le Quaternaire de la basse vallée de l'Aude. Bulletin de la Société Géologique de France, 12, p. 413-425.

Williams B., Walker J., Anderson J., 2006. Spatial variability of regolith leaching and salinity in relation to whole farm planning. Australian Journal of Experimental Agriculture, 46, p. 1271-1277. 
Figure 2 : Vivien MATHÉ et al., Prospections géophysiques multi-méthodes de structures portuaires antiques à Narbonne (Aude, France) (p. 52)
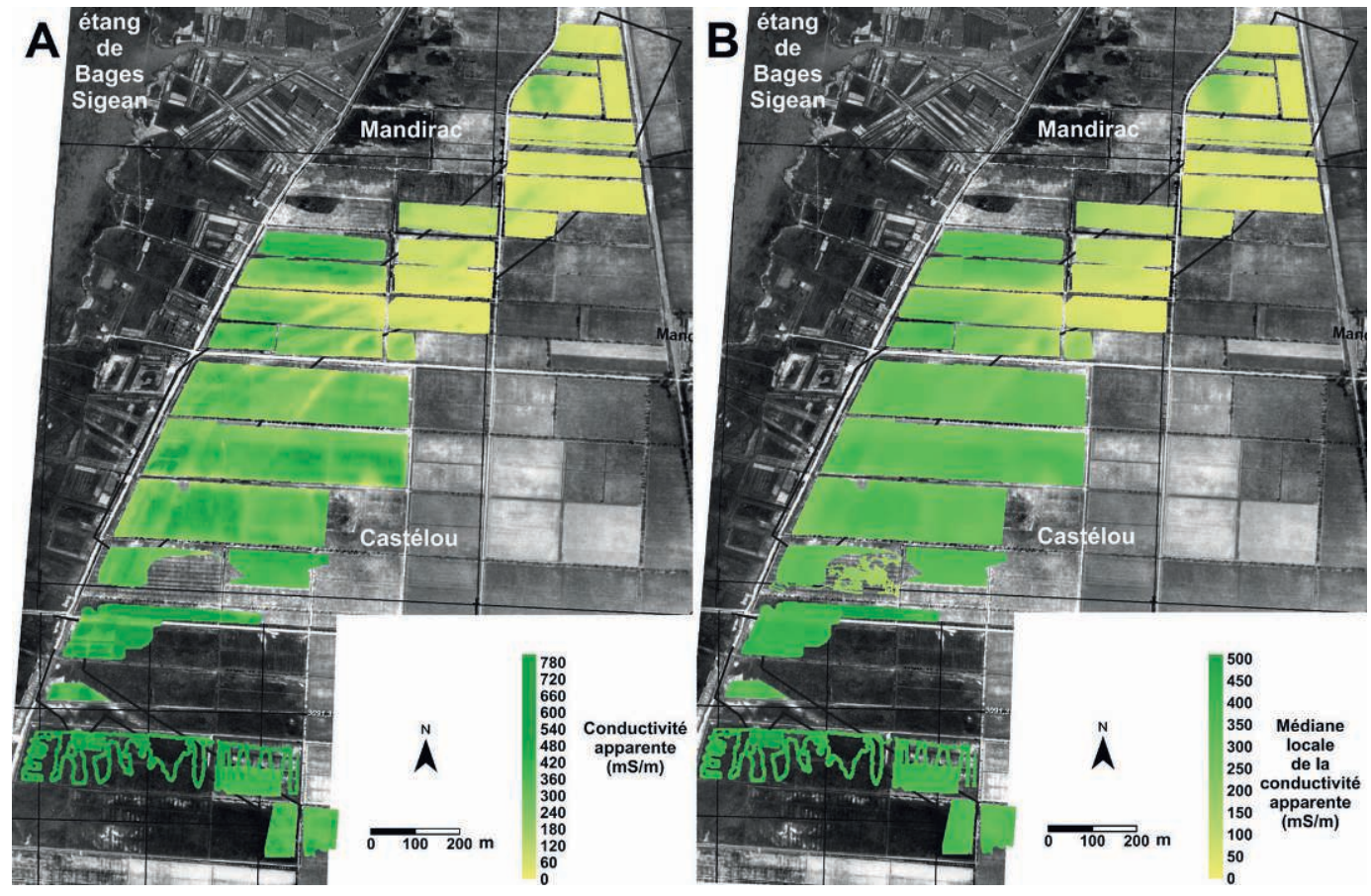

Figure 3 : Vivien Mathé et al., Prospections géophysiques multi-méthodes de structures portuaires antiques à Narbonne (Aude, France) (p. 53) 

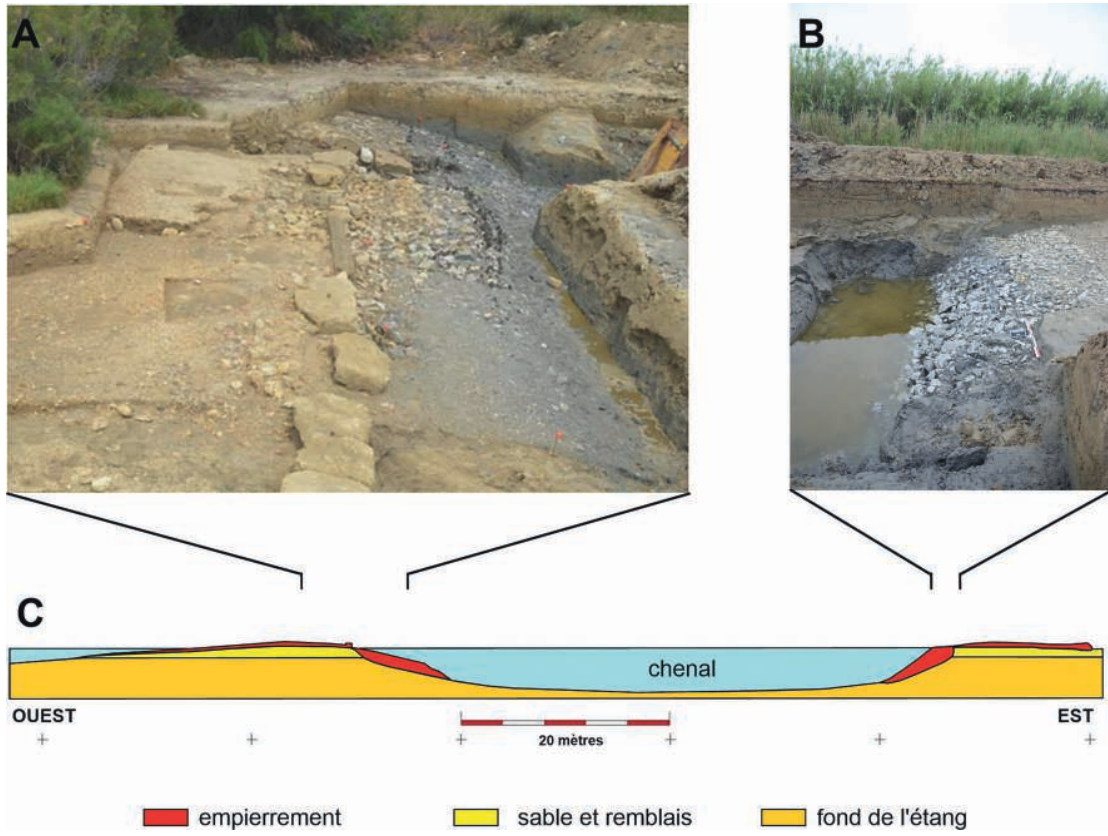

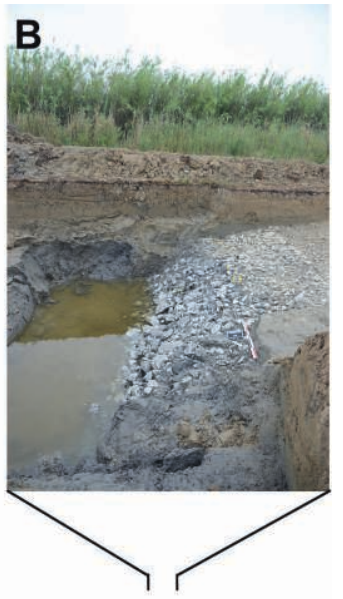

Figure 6 : Vivien Mathé et al., Prospections géophysiques multi-méthodes de structures portuaires antiques à Narbonne (Aude, France) (p. 55)
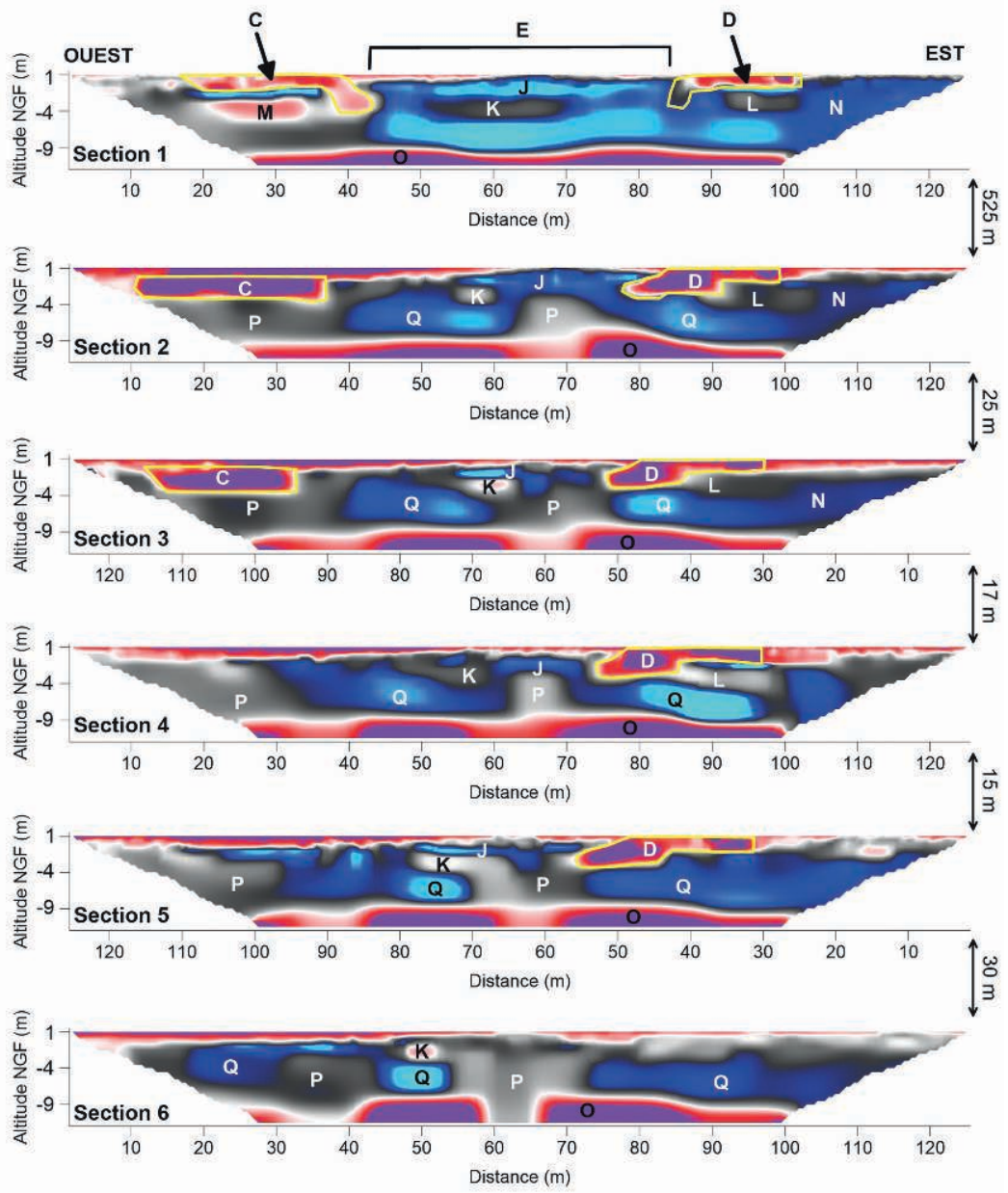

Figure 7 : Vivien Mathé et al., Prospections géophysiques multi-méthodes de structures portuaires antiques à Narbonne (Aude, France) (p. 56) 


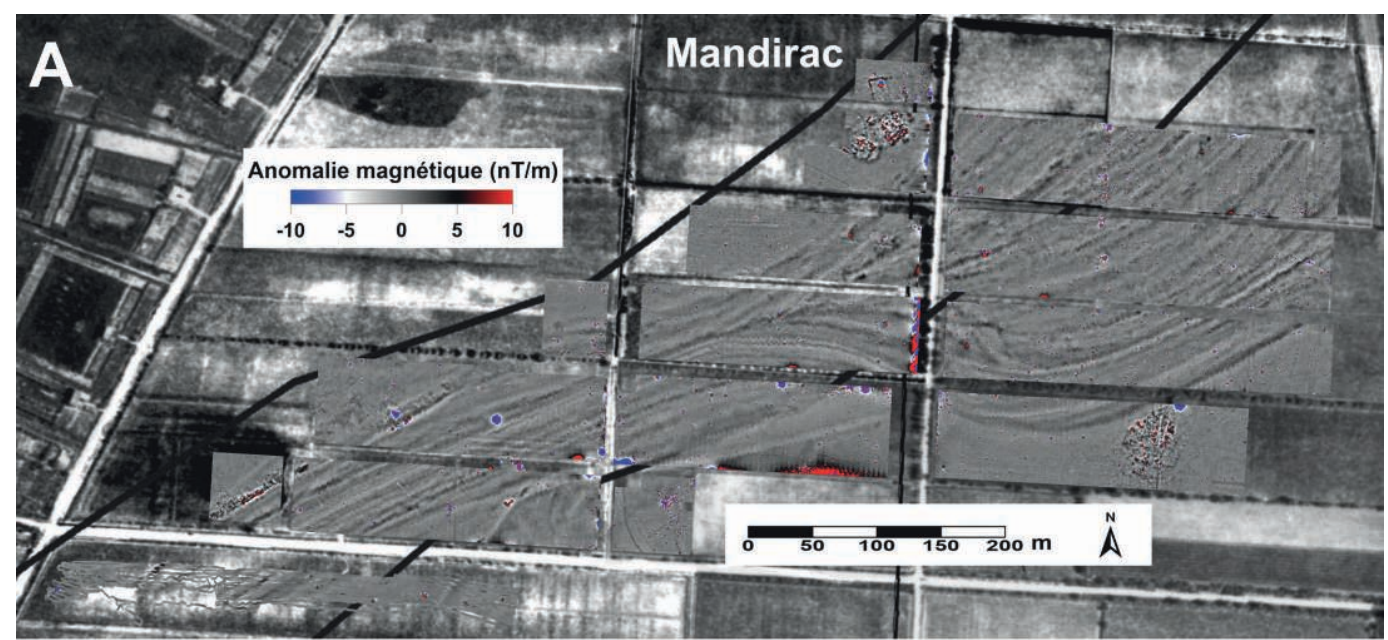

Figure 8 : Vivien Mathé et al., Prospections géophysiques multi-méthodes de structures portuaires antiques à Narbonne (Aude, France) (p. 57)
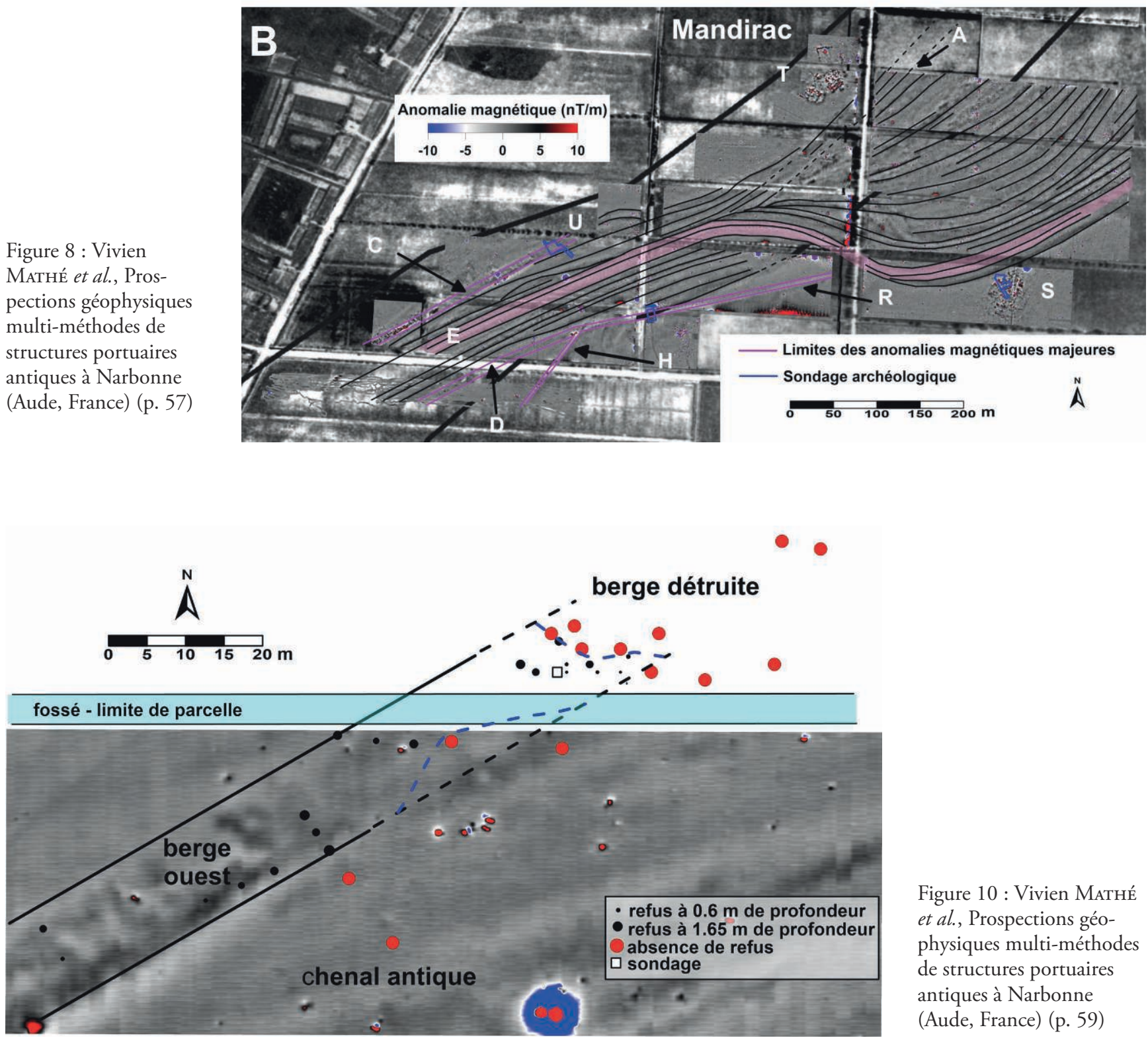

Figure 10 : Vivien Mathé et al., Prospections géophysiques multi-méthodes de structures portuaires antiques à Narbonne (Aude, France) (p. 59) 